TRANSACTIONS OF THE

AMERICAN MATHEMATICAL SOCIETY

Volume 352, Number 8, Pages 3743-3776

S 0002-9947(00)02543-5

Article electronically published on April 18, 2000

\title{
THE PROBLEM OF LACUNAS AND ANALYSIS ON ROOT SYSTEMS
}

\author{
YURI BEREST
}

\begin{abstract}
A lacuna of a linear hyperbolic differential operator is a domain inside its propagation cone where a proper fundamental solution vanishes identically. Huygens' principle for the classical wave equation is the simplest important example of such a phenomenon. The study of lacunas for hyperbolic equations of arbitrary order was initiated by I. G. Petrovsky (1945). Extending and clarifying his results, Atiyah, Bott and Gårding (1970-73) developed a profound and complete theory for hyperbolic operators with constant coefficients. In contrast, much less is known about lacunas for operators with variable coefficients. In the present paper we study this problem for one remarkable class of partial differential operators with singular coefficients. These operators stem from the theory of special functions in several variables related to finite root systems (Coxeter groups). The underlying algebraic structure makes it possible to extend many results of the Atiyah-Bott-Gårding theory. We give a generalization of the classical Herglotz-Petrovsky-Leray formulas expressing the fundamental solution in terms of Abelian integrals over properly constructed cycles in complex projective space. Such a representation allows us to employ the Petrovsky topological condition for testing regular (strong) lacunas for the operators under consideration. Some illustrative examples are constructed. A relation between the theory of lacunas and the problem of classification of commutative rings of partial differential operators is discussed.
\end{abstract}

\section{INTRODUCTION}

The classical integral formulas solving the initial boundary value problem for the wave equation

$$
\square_{n} u:=\left(\frac{\partial^{2}}{\partial x_{1}^{2}}-\frac{\partial^{2}}{\partial x_{2}^{2}}-\ldots-\frac{\partial^{2}}{\partial x_{n}^{2}}\right) u(x)=0, \quad x \in \mathbb{R}^{n},
$$

reveal a fundamental difference between even and odd $n$. When the number of dimensions is even $(n>2)$, Huygens' principle holds (cf. [33], [17]): the solution of a regular Cauchy problem for (0.1) at every point $x=x_{0}$ depends on its initial data only in an arbitrarily small neighborhood of the light cone surface with the vertex at this point. By contrast, for odd dimensions (and $n=2$ ), the corresponding domain of dependence also contains the interior points of the propagation cone.

Huygens' principle in that sense can be viewed as a prelude to the general theory of lacunas for hyperbolic differential operators of arbitrary order created by I. G. Petrovsky [50].

Received by the editors March 6, 1997 and, in revised form, November 3, 1997.

1991 Mathematics Subject Classification. Primary 58F07, 35L15; Secondary 58F37, 35L25.

Key words and phrases. Hyperbolic linear differential operators, fundamental solution, lacuna, Huygens' principle, Coxeter groups, Dunkl operators. 
According to Petrovsky's definition, a lacuna of a linear hyperbolic differential operator $\mathcal{L}$ is an open connected component $L$ of the complement of the conoid of bicharacteristics of $\mathcal{L}$, in which the principal fundamental solution $\Phi(\mathcal{L}, \cdot)$ vanishes identically.

In his paper [50] Petrovsky posed and studied the problem of lacunas mainly for homogeneous strongly hyperbolic operators with constant coefficients. One of the important results of his work was the discovery of a remarkable topological criterion for existence of lacunas for such operators (now referred to as the Petrovsky condition).

A further refinement of Petrovsky's theory and its generalization to the class of hyperbolic operators with multiple characteristics was accomplished by Atiyah, Bott and Gårding in their seminal treatise on lacunas 2, 23. In particular, these authors modified the notion of lacuna and proposed a relevant (local) version of Petrovsky's criterion 3 .

Lacunas in the sense of Atiyah, Bott and Gårding (usually called weak or $\mathcal{C}^{\infty}$ lacunas) are open connected components $L$ in the complement of a singular locus $W$ of the fundamental solution $\Phi(\mathcal{L}, \cdot)$, such that $\Phi(\mathcal{L}, \cdot)$ admits a local $\mathcal{C}^{\infty}$-extension from $L$ to its closure $\bar{L}$. In this case the distribution $\Phi(\mathcal{L}, \cdot)$ is said to have a sharp front from $L$ at each boundary point $x \in \partial L$.

Such a generalization made it possible to apply the methods of microlocal analysis and the theory of singularities to the closer study of weak lacunas and sharp fronts for hyperbolic operators both with constant and with variable (smooth) coefficients (see [27], [12], 28], [56], [57] and references therein).

Meanwhile, the problem of lacunas (in the original Petrovsky sense) for hyperbolic operators with variable coefficients seems to remain much less well-studied. Powerful pseudo-differential methods and, in particular, the theory of Fourier integral operators [38], [19] only provide an analysis of singularities in which one is led to neglect $\mathcal{C}^{\infty}$-functions, and thus they seem to be not quite adapted to investigation of lacunas in the strong sense.

To the best of author's knowledge 1 , the main results available in this field are concerned only with second order hyperbolic operators and related to the old question of Hadamard (see [33], book IV): Which wave-type operators satisfy Huygens' principle in its strict form?

In contrast with the higher order case, this problem is, in principle, accessible to local methods based on the Hadamard-Riesz type expansions of the fundamental distribution. Yet, in spite of considerable efforts, it is still far from completely solved (see, e.g., the monographs [17, 22, 32], surveys [23, [18, 37], [45], 10], as well as references therein).

Recently, a new class of second order hyperbolic operators compatible with Huygens' principle on Minkowski (flat) spaces has been found ([8, 9]). These operators can be viewed as a natural generalization of the classical examples due to Stellmacher ([52, 53]). Their construction, based on recent developments in the theory of special functions in several variables (see, e.g., [20], 21], [35], 36], [48, [49], [14], [15], [58]), reveals an interesting connection between Huygens' principle and Coxeter groups (i.e., finite reflection subgroups of the real orthogonal group $O(n))$.

\footnotetext{
${ }^{1}$ Cf. also remarks in [23], 18].
} 
The purpose of the present work is a further and deeper study of the link between the theory of lacunas and finite reflection groups. Our main concern will be to carry over the results of [8], [9] to the case of higher order linear hyperbolic differential operators

$$
\mathcal{L}=P(D)+\sum_{|\nu|<p} a_{\nu}(x) D^{\nu}, \quad D:=-i \frac{\partial}{\partial x},
$$

with constant principal symbols $P(\zeta), \operatorname{deg} P(\zeta)=p \geq 2$.

In fact, we give a generalization of the Petrovsky-Atiyah-Bott-Gårding theory to a class of hyperbolic operators with variable coefficients. The underlying algebraic structure makes it possible to represent the fundamental solutions of such operators in terms of Abelian integrals of Herglotz-Petrovsky type and then to employ the Petrovsky topological condition for testing their lacunas.

As should be clear a priori, the class of differential operators under consideration is quite exceptional. Physically, such operators occur as 'quantum Hamiltonians' of some completely integrable quantum models ([4]), while, mathematically, they stem from the theory of special functions in several variables (Bessel-type equations) related to Coxeter systems (see [49], 36] and references therein). Within the theory of partial differential equations these operators can be regarded as a far-reaching generalization of the classical Euler-Poisson-Darboux equations ([17]).

In a broader sense, we would like to view our results in the context of a natural extension of the Hadamard problem in a general setting of the theory of lacunas: For which hyperbolic polynomials $P \in \operatorname{Hyp}(\vartheta)$ do there exist nontrivial families of partial differential operators (0.2) having $P$ as a common principal symbol and admitting interior lacunas in their propagation cones? How can one characterize such families for a given $P(\zeta)$, if any do exist?

From the topological point of view, similar questions are raised and discussed in the recent book [57]. By contrast, our attempt here is to stress the algebrogeometric aspect of the problem and to explore some first instructive examples. The development of a complete classification theory seems hardly attainable at the present stage. Indeed, even in the classical case of the second order wave polynomial, the problem has been solved in dimension $n=4$ ([44], 34], 1] ), while in higher dimensions $(n \geq 6)$ a complete solution is available only for some restricted classes of wave-type operators (see [52, 41, [5]).

The paper is written in a self-contained manner 2 Our analysis rests heavily on the theory of lacunas for hyperbolic equations with constant coefficients. We have included the necessary results from this theory, following mostly [2], [3].

\section{RoOT SySTEMS AND ALGEBRAS OF DUNKL OPERATORS}

This section is a preliminary one. Here, we fix notation and prepare some auxiliary results (mostly of an algebraic nature) based on the work of Dunkl [20] and Heckman 35.

Let $V$ be a real finite-dimensional vector space $(\operatorname{dim} V=n)$ endowed with a positive definite symmetric bilinear form $(\cdot, \cdot)$. We write $V^{\prime}:=\operatorname{Hom}_{\mathbb{R}}(V, \mathbb{R})$ for its real dual, and $V_{\mathbb{C}}:=V \otimes \mathbb{C}, V_{\mathbb{C}}^{\prime}:=V^{\prime} \otimes \mathbb{C}$ for the corresponding complexifications. The following notation is conventional: $\mathbb{R}[V]$ is the ring of real polynomials on $V$,

${ }^{2}$ A brief summary of our main results can be found in $[$. 
and $\mathbb{C}[V]$ is its complex counterpart, a space of complex rational functions on $V$; End $[\mathbb{C}(V)]$ denotes the associative algebra of linear endomorphisms of $\mathbb{C}(V)$.

Consider a finite group $G \subset O(V)$ generated by (real) reflections in $V$. By a reflection we mean a linear invertible operator $s_{\alpha} \in \operatorname{End}(V)$ which sends some nonzero vector $\alpha \in V$ to its negative while fixing the orthogonal hyperplane $\alpha^{\perp}$ pointwise:

$$
s_{\alpha}=\mathrm{id}-\alpha \otimes \alpha^{\vee}, \quad \text { with } \quad \alpha^{\vee}:=2(\alpha, \alpha)^{-1}(\alpha, \cdot) \in V^{\prime} .
$$

Definition 1.1. A root system $\Re:=\Re(G)$ associated to $G$ is a finite set $\{\alpha\}$ of nonzero vectors in $V$ such that $(i) \Re \cap \mathbb{R} \Re= \pm \alpha,(i i) s_{\alpha}(\Re)=\Re$ for all $\alpha \in \Re$, and (iii) $G=\left\langle s_{\alpha} \mid \alpha \in \Re\right\rangle$.

Clearly, the system $\Re$ is determined up to a normalization of 'roots' $\alpha \in \Re$. The number $\operatorname{rk} G:=\operatorname{dim}(\operatorname{span} \Re)$ is called the $\operatorname{rank}$ of the group $G$. When $\operatorname{rk} G=n$, the group $G$ acts on $V$ with no (nonzero) fixed points and is referred to as essential ${ }^{3}$ We denote by $\Re_{+}$the subset of vectors in $\Re$ positive with respect to some total ordering in $V$. Then $\Re$ is obviously the disjoint union of $\Re_{+}$and $\left(-\Re_{+}\right)$.

Let $M:=M(G)$ be the linear space of all $\mathbb{R}$-valued $G$-invariant functions on $\Re, m: \Re \rightarrow \mathbb{R}, \alpha \mapsto m_{\alpha}$, called (root) multiplicities. The dimension of $M$ is equal to the number $r$ of $G$-orbits in $\Re$, which in turn is equal to the number of conjugacy classes of reflections in $G$.

Following Dunkl [20, we introduce a family of differential-reflection operators $\nabla_{m, v} \in \operatorname{End}[\mathbb{C}(V)]$ on the space $\mathbb{C}(V)$ parametrized by the elements of $M$. Namely, for fixed $v \in V$ and $m \in M$, we set

$$
\nabla_{m, v}:=\partial_{v}+\sum_{\alpha \in \Re_{+}} m_{\alpha} \frac{(\alpha, v)}{(\alpha, \cdot)} \hat{s}_{\alpha},
$$

where $\partial_{v}$ stands for the derivative in direction $v,(\alpha, \cdot) \in V^{\prime} \subset \mathbb{C}[V]$ is the linear form $x \mapsto(\alpha, x)$ on $V$, and the hat over $g \in G$ denotes the standard representation of $G$ on $\mathbb{C}(V)$, i.e. $\hat{g}: f(x) \mapsto f\left(g^{-1}(x)\right), f(\cdot) \in \mathbb{C}(V)$.

Remark 1.2. Dealing with polynomial (rather than rational) functions on $V$, Dunkl defines his operators in a different (but essentially equivalent to (1.2) form (cf. 20]).

Remark 1.3. The definition (1.2) is independent of the length of roots in $\Re$ and the choice of total ordering in $V$. Indeed, since $m_{\alpha}=m_{-\alpha}$, we have

$$
\nabla_{m, v}:=\partial_{v}+\frac{1}{2} \sum_{\alpha \in \Re} m_{\alpha} \frac{(\alpha, v)}{(\alpha, \cdot)} \hat{s}_{\alpha} .
$$

The family of Dunkl operators is determined by the finite reflection group $G$ and the multiplicity function $m \in M(G)$.

The basic properties of the operators (1.2) are given in the following lemma.

Lemma 1.4 (Dunkl, 20]). For all $u, v \in V, m \in M$ and $g \in G$, the following properties hold:

(a) commutativity:

$$
\nabla_{m, u} \circ \nabla_{m, v}=\nabla_{m, v} \circ \nabla_{m, u} .
$$

\footnotetext{
${ }^{3}$ In the sequel, we will sometimes allow $\operatorname{rk} G<n$.
} 
(b) G-equivariance:

$$
\hat{g} \circ \nabla_{m, v} \circ \hat{g}^{-1}=\nabla_{m, g(v)} .
$$

(c) homogeneity: When $f \in \mathbb{C}_{p}(V)$ is homogeneous of degree $p, \nabla_{m, v}[f]$ is homogeneous of degree $p-1$.

The commutativity condition $(a)$ is verified by a straightforward calculation. In fact, the terms with derivatives are cancelled identically in (1.3), and the commutator $\left[\nabla_{m, u}, \nabla_{m, v}\right]$ vanishes if and only if the following identity holds:

$$
\sum_{\alpha, \beta \in \Re_{+}} m_{\alpha} m_{\beta} \frac{[(\alpha, u)(\beta, v)-(\alpha, v)(\beta, u)]}{(\alpha, \cdot)\left(s_{\alpha}(\beta), \cdot\right)} \hat{s}_{\alpha} \hat{s}_{\beta}=0 .
$$

The latter is known to be valid for any Coxeter root system (see [20], Proposition 1.7, and 35], Proposition 2.2). The properties $(b)$ and $(c)$ follow immediately from the definiton (1.2).

Let $\mathbb{C}\left[V^{\prime}\right] \cong \operatorname{Sym}_{\mathbb{C}}(V)$ be the (complexified) symmetric algebra over $V$. According to Lemma 1.4 for any fixed $m \in M$ we may define an algebra homomorphism $\nabla_{m}: \mathbb{C}\left[V^{\prime}\right] \rightarrow \operatorname{End}[\mathbb{C}(V)]$ sending each monomial $v_{1}^{\nu_{1}} \cdots v_{p}^{\nu_{p}}, v_{i} \in V, \nu_{i} \in \mathbb{Z}_{+}$, to $\nabla_{m, v_{1}}^{\nu_{1}} \circ \cdots \circ \nabla_{m, v_{p}}^{\nu_{p}}$ and extending the result to all of $\mathbb{C}\left[V^{\prime}\right]$ by linearity. The image of $\nabla_{m}$, denoted by $\mathbb{C}\left[\nabla_{m}\right]$, can be viewed as a commutative associative deformation of the ring of constant coefficient differential operators on $V$.

Since the homomorphism $\nabla_{m}$ is injective, the standard 'diagonal' representation of $G, G \rightarrow \operatorname{Aut}\left(\mathbb{C}\left[V^{\prime}\right]\right)$, carries over to its image $\mathbb{C}\left[\nabla_{m}\right]$. The proper subalgebra of $\mathbb{C}\left[\nabla_{m}\right]$ generated by all $G$-invariant operators will be then denoted by $\mathbb{C}\left[\nabla_{m}\right]^{G}$.

The following lemma will be basic for the study of analytic properties of (generalized) Riesz kernels in Section 2.3.

Lemma 1.5. Let $\mathcal{W}_{m} \subset \operatorname{End}[\mathbb{C}(V)]$ be the associative algebra generated by (multiplication by) $v_{*}:=(v, \cdot)$ and $\nabla_{m, u}$ for $v, u \in V$. Then, the adjoint representation of the invariant commutative subalgebra $\mathbb{C}\left[\nabla_{m}\right]^{G} \subset \mathcal{W}_{m}$ is locally nilpotent on $\mathcal{W}_{m}$. More precisely, for any $G$-invariant operator $\nabla_{m}(P) \in \mathbb{C}\left[\nabla_{m}\right]^{G}$ and for any $T \in \mathcal{W}_{m}$,

$$
\operatorname{ad}_{\nabla_{m}(P)}^{N}[T]=0 \text { with some } N \in \mathbb{Z}_{+} .
$$

Proof. The statement follows from the commutativity property of Dunkl operators (see Lemma $1.4(a)$ ) and the identity

$$
\operatorname{ad}_{\nabla_{m}(P)}\left[v_{*}\right]=\nabla_{m}\left(\partial_{v_{*}} P\right),
$$

valid for arbitrary $G$-invariant $P \in \mathbb{C}\left[V^{\prime}\right]^{G}$. It remains to check (1.6).

Let $Q:=\sum_{i=1}^{n} e_{i} \otimes e_{i} \in \mathbb{C}\left[V^{\prime}\right]^{G}$ for some orthonormal basis $e_{1}, \ldots, e_{n}$ in $V$, and denote by $\Delta_{m}:=\nabla_{m}(Q)$ its image in $\mathbb{C}\left[\nabla_{m}\right]^{G}$. Then, (1.6) is easily verified for $Q$ by a straightforward calculation:

$$
\left[\Delta_{m}, v_{*}\right]=2 \nabla_{m, v},
$$

and, hence,

$$
\operatorname{ad}_{\Delta_{m}}^{2}\left[v_{*}\right]=0 .
$$

It follows from (1.7) and (1.8) that

$$
\nabla_{m}\left(P_{k}\right)=\frac{1}{2^{k} k !} \operatorname{ad}_{\Delta_{m}}^{k}\left[P_{k}\right]
$$


for arbitrary $P_{k} \in \mathbb{C}\left[V^{\prime}\right] \cong \mathbb{C}[V]$ homogeneous of degree $k$ (cf. [35], Proposition 3.4). Here, $\mathbb{C}\left[V^{\prime}\right]$ and $\mathbb{C}[V]$ are identified with the help of the invariant inner product $(\cdot, \cdot)$ on $V$.

Representing $P \in \mathbb{C}\left[V^{\prime}\right]^{G}$ as a sum of $G$-invariant homogeneous components $P=\sum_{k \geq 0} P_{k}$, and repeatedly using (1.9), (1.8) and (1.7), we finally obtain

$$
\begin{aligned}
\operatorname{ad}_{\nabla_{m}(P)}\left[v_{*}\right] & =-\sum_{k \geq 0} \operatorname{ad}_{v_{*}}\left[\nabla_{m}\left(P_{k}\right)\right] \\
& =-\sum_{k \geq 0} \frac{1}{2^{k} k !} \operatorname{ad}_{v_{*}} \circ \operatorname{ad}_{\Delta_{m}}^{k}\left[P_{k}\right] \\
& =-\sum_{k \geq 0} \frac{k}{2^{k} k !} \operatorname{ad}_{\Delta_{m}}^{k-1} \circ \operatorname{ad}_{\left[v_{*}, \Delta_{m}\right]}\left[P_{k}\right] \\
& =\sum_{k \geq 1} \frac{1}{2^{k-1}(k-1) !} \operatorname{ad}_{\Delta_{m}}^{k-1}\left[\partial_{v_{*}} P_{k}\right]=\nabla_{m}\left(\partial_{v_{*}} P\right)
\end{aligned}
$$

Let Char $(G)$ be the multiplicative group of (sign) characters $\varepsilon: G \rightarrow \mathbb{Z}_{2}, g \mapsto$ $\operatorname{det} g$, of the group $G$. We associate to each $\varepsilon \in \operatorname{Char}(G)$ a $G$-invariant subset of roots $\mathcal{E} \subset \Re$ in such a way that

$$
\mathcal{E}:=\left\{\alpha \in \Re \mid \varepsilon\left(s_{\alpha}\right)=-1\right\} .
$$

The characteristic function of $\mathcal{E}$ in $\Re$ is denoted by $1_{\varepsilon}: \Re \rightarrow\{0,1\}$.

Consider $\mathbb{C}[V]_{\varepsilon}^{G} \subset \mathbb{C}[V]$, the space of relative polynomial invariants of $G$ associated with the character $\varepsilon$. When $\varepsilon$ is trivial, i.e. $\varepsilon \equiv 1, \mathbb{C}[V]_{\varepsilon}^{G}:=\mathbb{C}[V]^{G}$ is a (free) invariant algebra generated by $n$ homogeneous polynomials of some fixed degrees (Chevalley's theorem), while, for arbitrary $\varepsilon \in \operatorname{Char}(G), \mathbb{C}[V]_{\varepsilon}^{G}$ is the (free) module of rank one over $\mathbb{C}[V]^{G}$ generated by the proper alternating polynomial

$$
\theta_{\varepsilon}:=\prod_{\alpha \in \Re_{+}} \alpha_{*}^{1_{\varepsilon}(\alpha)}=\prod_{\alpha \in \mathcal{E}_{+}} \alpha_{*}, \quad \mathcal{E}_{+}:=\mathcal{E} \cap \Re_{+} .
$$

Let $V_{\text {reg }}^{\mathbb{C}}:=V_{\mathbb{C}} \backslash \bigcup \alpha_{\mathbb{C}}^{\frac{1}{}}$ be an open (quasi-)affine algebraic variety ${ }^{\mathbb{4}}$ in $V_{\mathbb{C}} \cong \mathbb{C}^{n}$ obtained by removing the (complexified) reflection hyperplanes of the group $G$. We write $\mathcal{O}:=\mathcal{O}\left(V_{\text {reg }}^{\mathbb{C}}\right)$ for the coordinate ring of regular functions and $\mathfrak{D}:=\mathfrak{D}\left(V_{\text {reg }}^{\mathbb{C}}\right)$ for the ring of (algebraic) differential operators on $V_{\text {reg. }}^{\mathbb{C}}$. Note that both $\mathcal{O}$ and $\mathfrak{D}$ can be identified respectively with the localizations of the polynomial ring $\mathbb{C}[V]$ and the Weyl algebra $\mathbb{A}_{n}(\mathbb{C}):=\mathbb{C}\left\langle x, \frac{\partial}{\partial x}\right\rangle$ at the fundamental alternating polynomial $\theta:=\prod_{\alpha \in \Re_{+}} \alpha_{*}$, i. e.

$$
\mathcal{O} \cong \mathbb{C}\left[V, \theta^{-1}\right], \quad \mathfrak{D} \cong \mathbb{C}\left[V, \theta^{-1}\right] \otimes_{\mathbb{C}[V]} \mathbb{A}_{n}(\mathbb{C}) .
$$

Since $V_{\text {reg }}^{\mathbb{C}}$ is a $G$-invariant subvariety in $V_{\mathbb{C}}$, the action of the group $G$ on $\mathbb{C}[V]$ can be extended to $\mathcal{O}$; the space of (semi-)invariant regular functions on $V_{\text {reg }}^{\mathbb{C}}$ associated with a character $\varepsilon \in \operatorname{Char}(G)$ will then be denoted by $\mathcal{O}_{\varepsilon}^{G}$.

\footnotetext{
${ }^{4}$ The notation $V_{\text {reg }}$ is preserved for the set of all $\mathbb{R}$-rational points of $V_{\text {reg }}^{\mathbb{C}}$.
} 
Let $\varepsilon \in \operatorname{Char}(G)$ and $m \in M$ be fixed. Following [35], we define the (restriction) map $\operatorname{Res}^{\varepsilon}: \mathcal{W}_{m} \rightarrow \mathfrak{D}$ by the following characteristic property: if $T \in \mathcal{W}_{m}$, then

$$
\left(\operatorname{Res}^{\varepsilon} T\right)[f]=T[f] \quad \text { whenever } f \in \mathcal{O}_{\varepsilon}^{G} \text {. }
$$

It is easy to see that $\operatorname{Res}^{\varepsilon}$ is a uniquely determined operation which associates to a (nonlocal) operator $T \in \mathcal{W}_{m} \subset \operatorname{End}[\mathbb{C}(V)]$ its purely differential part $\operatorname{Res}^{\varepsilon} T \in \mathfrak{D}$ depending on the character $\varepsilon \in \operatorname{Char}(G)$. Indeed, given any element $T=\sum a_{\nu_{1} \cdots \nu_{n}} \nabla_{m, v_{1}}^{\nu_{1}} \cdots \nabla_{m, v_{n}}^{\nu_{n}} \in \mathbb{C}\left[\nabla_{m}\right]$, the restriction $\operatorname{Res}^{\varepsilon} T, \varepsilon \in \operatorname{Char}(G)$, can be evaluated effectively. For this, using the obvious relations

$$
\hat{s}_{\alpha} \circ \partial_{v}=\partial_{s_{\alpha}(v)} \circ \hat{s}_{\alpha}, \quad \hat{s}_{\alpha} \circ f=\hat{s}_{\alpha}(f) \circ \hat{s}_{\alpha}, \quad f \in \mathcal{O},
$$

one has to carry over the reflection operators in each monomial of $T$ to the right and then to replace $\hat{s}_{\alpha}$ by a proper character value $\varepsilon\left(s_{\alpha}\right)$. For example, we have

$$
\operatorname{Res} \Delta_{m}=\sum_{i=1}^{n} \partial_{e_{i}}^{2}-\sum_{\alpha \in \Re_{+}} \frac{m_{\alpha}\left(m_{\alpha}+1\right)(\alpha, \alpha)}{(\alpha, x)^{2}}
$$

for $\Delta_{m} \in \mathbb{C}\left[\nabla_{m}\right]^{G}$ defined in the proof of Lemma 1.5. (When $\varepsilon$ is trivial, we usually omit the superscript $\varepsilon$ in the notation $\operatorname{Res}^{\varepsilon}$.)

The following lemma is an immediate consequence of the definition of $\operatorname{Res}^{\varepsilon}$.

Lemma 1.6. Let $\mathcal{W}_{m, \varepsilon}^{G}$ be the (linear) subspace in $\mathcal{W}_{m}$ generated by $\varepsilon$-invariant operators:

$$
\mathcal{W}_{m, \varepsilon}^{G}:=\left\{T \in \mathcal{W}_{m} \mid \operatorname{Ad}_{g}(T)=\varepsilon(g) T, g \in G\right\} .
$$

Then, if $T \in \mathcal{W}_{m}$ and $B \in \mathcal{W}_{m, \varepsilon}^{G}$, we have

$$
\operatorname{Res}^{\varepsilon^{\prime}}(T B)=\operatorname{Res}^{\varepsilon^{\prime} \varepsilon}(T) \operatorname{Res}^{\varepsilon^{\prime}}(B) \quad \text { for any } \varepsilon^{\prime} \in \operatorname{Char}(G) .
$$

Theorem 1.7 (Heckman, [35]). Let $\mathfrak{D}^{G, m} \subset \mathfrak{D}$ be the image of $\mathbb{C}\left[\nabla_{m}\right]^{G} \subset \mathcal{W}_{m}^{G}$ under the restriction map related to the trivial character, i.e.

$$
\mathfrak{D}^{G, m}:=\operatorname{Res}\left(\mathbb{C}\left[\nabla_{m}\right]^{G}\right), \quad m \in M(G) .
$$

Then, $\mathfrak{D}^{G, m}$ is a commutative ring of regular differential operators in $\mathfrak{D}$ isomorphic to the algebra of $G$-invariant polynomials $\mathbb{C}\left[V^{\prime}\right]^{G}$.

The proof of this theorem follows directly from Lemma 1.4 (a) and Lemma 1.6 (use formula (1.14) with $\varepsilon=\varepsilon^{\prime} \equiv 1$ ).

\section{INVARIANT HYPERBOLIC OPERATORS ON ROOT SYSTEMS}

In this section we single out a multiplicative semigroup of hyperbolic differential operators in the commutative ring $\mathfrak{D}^{G, m}$ and study analytic properties of associated Riesz kernels.

2.1. Hyperbolic polynomials. Recall first some basic definitions from the theory of hyperbolic operators with constant coefficients [26] (see also [2]-3] and [39], vol. II).

Let $V$ and $V^{\prime}$ be a pair of dual vector spaces (as above) equipped with canonical affine structures. We fix biorthogonal coordinates $x=\left(x^{1}, \ldots, x^{n}\right), \zeta=$ $\left(\zeta^{1}, \ldots, \zeta^{n}\right)$ on $V$ and $V^{\prime}$, and identify $\mathbb{C}[V]$ and $\mathbb{C}\left[V^{\prime}\right]$ with the standard polynomial rings $\mathbb{C}[x]$ and $\mathbb{C}[\zeta]$ respectively.

Let $P \in \mathbb{C}\left[V^{\prime}\right]$, and let $P_{0}$ stand for the principal part of $P$. 
Definition 2.1 (Gårding, 26]). The polynomial $P(\zeta)$ is called hyperbolic in the direction of a real vector $\vartheta \in V^{\prime}$ if $P_{0}(\vartheta) \neq 0$, and $P(\xi+\vartheta t) \neq 0$ for all $\xi \in V^{\prime}$ and for all $t \in \mathbb{C}$ with $|\operatorname{Im} t|$ large enough.

The set of all hyperbolic polynomials in the direction $\vartheta$ is denoted by $\operatorname{hyp}(\vartheta) \subset$ $\mathbb{C}\left[V^{\prime}\right]$. The homogeneous polynomials $P(\zeta) \equiv P_{0}(\zeta)$ in hyp $(\vartheta)$ are characterized by the simple condition

$$
\xi \in V^{\prime}, \operatorname{Im} t \neq 0 \Rightarrow P(\xi+\vartheta t) \neq 0,
$$

or, equivalently, the algebraic equation $P(\xi+\vartheta t)=0$ has $p=\operatorname{deg} P$ real roots with respect to $t$ for each $\xi \in V^{\prime}$. In addition, if these roots are pairwise distinct, unless $\xi$ is proportional to $\vartheta, P(\zeta)$ is called strongly hyperbolic (or hyperbolic in the sense of Petrovsky).

In the sequel, we will deal mainly with homogeneous hyperbolic polynomials. We write $\operatorname{Hyp}(\vartheta, p)$ for the space of such polynomials of degree $p$, and $\operatorname{Hyp}(\vartheta):=$ $\bigcup_{p \geq 0} \operatorname{Hyp}(\vartheta, p)$. Clearly, $\operatorname{Hyp}(\vartheta)=\operatorname{Hyp}(-\vartheta)$. In view of (2.1),$P(\zeta) / P(\vartheta)$ should be real when $P(\zeta) \in \operatorname{Hyp}(\vartheta)$. Hence, if necessary, we may restrict $\operatorname{Hyp}(\vartheta)$ to real polynomials, $\operatorname{Hyp}_{\mathbb{R}}(\vartheta):=\operatorname{Hyp}(\vartheta) \cap \mathbb{R}\left[V^{\prime}\right]$, without loss of generality.

The following lemmas collect some topological and algebraic properties of the space $\operatorname{Hyp}(\vartheta)$ (or rather $\left.\operatorname{Hyp}_{\mathbb{R}}(\vartheta)\right)$.

Lemma 2.2 (Nuij, 46]). The set $\operatorname{Hyp}_{\mathbb{R}}^{\circ}(\vartheta, p) \subset \operatorname{Hyp}_{\mathbb{R}}(\vartheta, p)$ of strongly hyperbolic polynomials is open in the space $\mathbb{R}_{p}\left[V^{\prime}\right]$ of all real homogeneous polynomials of degree $p, \operatorname{Hyp}_{\mathbb{R}}(\vartheta, p) \subset \overline{\operatorname{Hyp}_{\mathbb{R}}^{\circ}(\vartheta, p)}$ being the part of the closure of this set where $P(\vartheta) \neq 0$.

Lemma 2.3 (Gårding, [26]). The space $\operatorname{Hyp}(\vartheta)$ is closed under the following operations:

(a) multiplication:

$$
P_{1}, P_{2}, \ldots \in \operatorname{Hyp}(\vartheta) \quad \Rightarrow \quad P_{1} P_{2} \cdots \in \operatorname{Hyp}(\vartheta) .
$$

(b) factorization:

$$
\operatorname{Hyp}(\vartheta) \ni P=P_{1} P_{2} \cdots \quad \Rightarrow \quad P_{1}, P_{2}, \ldots \in \operatorname{Hyp}(\vartheta) .
$$

(c) polarization: If $P \in \operatorname{Hyp}(\vartheta, p)$ and $P_{k}(\zeta) \in \mathbb{C}\left[V^{\prime}\right]$ are polarizations of $P$ in the direction of $\vartheta$, i.e. the coefficients of the polynomial $P(\zeta+\vartheta t)=\sum t^{k} P_{k}(\zeta)$ under ascending powers of $t$, then $P_{k} \in \operatorname{Hyp}(\vartheta, p-k), k=0, \ldots, p$.

(d) localization: If $P \in \operatorname{Hyp}(\vartheta), \xi \in V^{\prime}$, and $P_{\xi}(\zeta) \in \mathbb{C}\left[V^{\prime}\right]$ is a localization of $P$ at $\xi$, i.e. the lowest nonzero term of the polynomial

$$
t \mapsto P(\xi+t \zeta)=t^{\mu_{\xi}} P_{\xi}(\zeta)+\mathcal{O}\left(t^{\mu_{\xi}+1}\right), \quad \mu_{\xi}=\operatorname{deg} P_{\xi},
$$

then $P_{\xi} \in \operatorname{Hyp}\left(\vartheta, \mu_{\xi}\right)$.

For the proof of Lemma 2.2 see 46 . The properties stated in Lemma 2.3 follow essentially from the definition of hyperbolicity (2.1). The condition (2.2) implies that $\operatorname{Hyp}(\vartheta)$ is a multiplicative semigroup in the ring $\mathbb{C}\left[V^{\prime}\right]$.

Let $P(\zeta) \in \operatorname{Hyp}(\vartheta)$. Consider the algebraic hypersurface of real zeros of $P$ :

$$
\Xi:=\left\{\xi \in V^{\prime} \mid P(\xi)=0\right\} \text {. }
$$

Typically, the projective image of $\Xi$ in $\mathbb{R P}^{n-1}$ consists of $[p / 2]$ possibly intersecting real ovals embedded one into another and, in case of odd $p$, an extra projective surface called 'the unpaired piece' (see Fig. 1). 


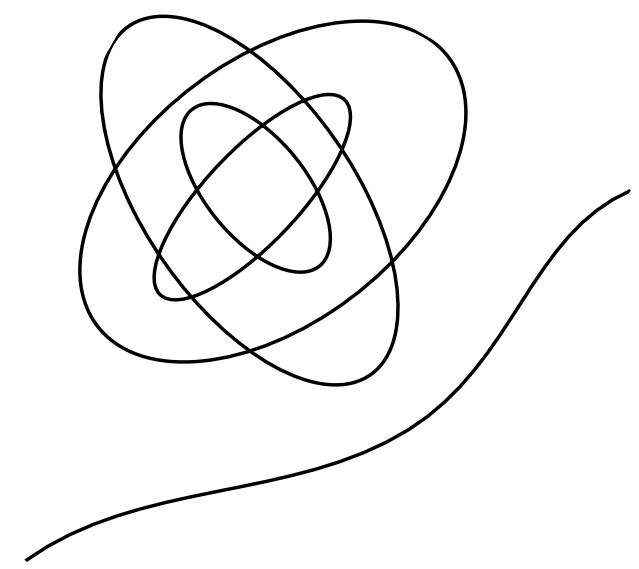

Figure 1. A real hyperbolic curve in $\mathbb{R}^{2}$

The open connected component $\Gamma(P, \vartheta)$ of the real complement $V^{\prime} \backslash \Xi$ of $\Xi$, containing the vector $\vartheta$, is called the hyperbolicity cone of $P$. It is a basic property of a hyperbolic polynomial $P \in \operatorname{Hyp}(\vartheta)$ that $\Gamma(P, \vartheta)$ is a convex cone, and $P \in \operatorname{Hyp}(\eta)$ for any $\eta \in \Gamma(P, \vartheta)$.

The dual cone for $\Gamma(P, \vartheta)$ with the vertex at a point $x_{0} \in V$,

$$
K\left(P, \vartheta, x_{0}\right):=\left\{x \in V \mid\left(\eta, x-x_{0}\right) \geq 0, \forall \eta \in \Gamma(P, \vartheta)\right\},
$$

is a closed convex affine set in $V$, referred to as the propagation cone of $P$. We simply write $K(P, \vartheta)$ instead of (2.6), when $x_{0}$ is not specified or $x_{0}=0$.

When $P(\zeta)$ has a trivial lineality 5 i.e. $P_{\xi}(\zeta) \equiv P(\zeta)$ implies $\xi=0$, the cone $\Gamma(P, \vartheta)$ is proper (peaked) in the sense that $\overline{\Gamma(P, \vartheta)}$ does not contain any straight lines, and then $K\left(P, \vartheta, x_{0}\right)$ has a non-empty interior $K^{\circ}\left(P, \vartheta, x_{0}\right)$. More generally, let $\Lambda(P)$ be a real lineality of $P(\zeta) \in \operatorname{Hyp}(\vartheta)$, i.e. the maximal linear subspace of $V^{\prime}$ such that $P$ can be restricted to a polynomial on the quotient $V^{\prime} / \Lambda(P)$. Then $\Lambda(P)$ coincides with the edge of the hyperbolicity cone $\Gamma(P, \vartheta)$, so that $\Gamma+\Lambda=\Gamma$, and $K(P, \vartheta)$ spans its orthogonal complement $\Lambda^{\perp}(P)$ in $V$.

Let $P \in \operatorname{Hyp}(\vartheta)$, and let $\xi \in V^{\prime}$ be fixed. Consider the localization $P_{\xi}$ of $P$ at $\xi$. By Lemma $2.3(d), P_{\xi} \in \operatorname{Hyp}(\vartheta)$, and we may define the local hyperbolicity and the local propagation cones of $P$ at $\xi$ by setting, respectively,

$$
\Gamma_{\xi}(P, \vartheta):=\Gamma\left(P_{\xi}, \vartheta\right), \quad K_{\xi}\left(P, \vartheta, x_{0}\right):=K\left(P_{\xi}, \vartheta, x_{0}\right) .
$$

Clearly, $\Gamma_{\xi}(P, \vartheta) \supseteq \Gamma(P, \vartheta)$ and, hence, $K_{\xi}\left(P, \vartheta, x_{0}\right) \subseteq K\left(P, \vartheta, x_{0}\right)$ for all $\xi \in V^{\prime}$. More precisely, the mapping $(\xi, P) \mapsto \Gamma_{\xi}(P, \vartheta)$ (and $(\xi, P) \mapsto K_{\xi}(P, \vartheta)$ ) is inner (resp., outer) continuous in the sense that $\Gamma_{\xi}(P, \vartheta) \cap \Gamma_{\tilde{\xi}}(\tilde{P}, \vartheta)$ (resp., $K_{\xi}(P, \vartheta) \cup$ $\left.K_{\tilde{\xi}}(\tilde{P}, \vartheta)\right)$ is close to $\Gamma_{\xi}(P, \vartheta)$ (resp., $K_{\xi}(P, \vartheta)$ ) when $(\tilde{\xi}, \tilde{P})$ is close to $(\xi, P)$ with $\xi, \tilde{\xi} \in V^{\prime}$ and $P, \tilde{P} \in \operatorname{Hyp}(\vartheta)$.

\footnotetext{
${ }^{5}$ Such polynomials are called complete since they depend essentially on all $n$ variables.
} 
The wave front surface $W$ of a hyperbolic polynomial $P \in \operatorname{Hyp}(\vartheta)$ is generated by the union of local propagation cones:

$$
W\left(P, \vartheta, x_{0}\right):=\bigcup_{0 \neq \xi \in V^{\prime}} K_{\xi}\left(P, \vartheta, x_{0}\right) .
$$

When $P$ is complete, i.e. $\Lambda(P)=\{0\}, W(P, \vartheta)$ is a closed semi-algebraic part of the global propagation cone $K(P, \vartheta)$ containing its boundary. More precisely,

$$
\partial K \subseteq W \subseteq K \cap \Xi^{\prime},
$$

where $\Xi^{\prime}:=\bigcup \Lambda^{\perp}\left(P_{\xi}\right), \xi \in V^{\prime} \backslash\{0\}$, is a real dual of the hyperbolic hypersurface $\Xi$. If $P \in \operatorname{Hyp}^{\circ}(\vartheta)$ and $\Xi$ is regular outside the origin, every nonzero $\xi \in \Xi^{\prime}$ admits only a one-dimensional space $\Lambda^{\perp}\left(P_{\xi}\right)$ of real normals, $n_{\xi}(P):=\operatorname{dim} \Lambda^{\perp}\left(P_{\xi}\right)=1$, half of it being $K_{\xi}(P, \vartheta)$. In that case, we have the equality $W=K \cap \Xi^{\prime}$. Otherwise, when $\Xi$ has singular points $\xi \neq 0$ with normals of a higher dimension, $n_{\xi}(P)>1$, the surface $W$ may be strictly smaller than $K \cap \Xi^{\prime}$. However, $\operatorname{codim} W(P, \vartheta)=1$ in any case, since $\xi \in \Lambda\left(P_{\xi}\right)$ and, hence, each $K_{\xi}\left(P, \vartheta, x_{0}\right)$ in (2.8) lies in a proper affine hyperplane normal to $\xi \neq 0$. Note also that, unlike $\Xi^{\prime}, W(P, \vartheta)$ depends on $P$ (outer) continuously.

For incomplete polynomials $P \in \operatorname{Hyp}(\vartheta)$ with $\Lambda(P) \neq\{0\}$, the wave front surface $W(P, \vartheta)$ equals $K(P, \vartheta)$, as $P_{\xi}(\zeta) \equiv P(\zeta)$ for each $\xi \in \Lambda(P)$, and, hence, is not the proper object to consider. Instead, one might define $W(P, \vartheta)$ by formula (2.8) with union over $\xi \notin \Lambda(P)$. Then restriction to the quotient $V^{\prime} / \Lambda(P)$ would reduce the situation to the previous case. In what follows we will therefore assume $P \in \operatorname{Hyp}(\vartheta)$ to be complete except where otherwise stated.

2.2. Hyperbolic operators in $\mathfrak{D}^{G, m}$. Let $G$ be a finite reflection group in $V$ of rank $\operatorname{rk} G \leq n$. Fix a real nonzero vector $\vartheta \in V^{\prime}$ and consider the set of homogeneous polynomials $\operatorname{Hyp}(\vartheta) \subset \mathbb{C}\left[V^{\prime}\right]$ hyperbolic in direction $\vartheta$. The subset of polynomials in $\operatorname{Hyp}(\vartheta)$ invariant under the natural representation of $G$ will be denoted by

$$
\operatorname{Hyp}(\vartheta)^{G}:=\operatorname{Hyp}(\vartheta) \cap \mathbb{C}\left[V^{\prime}\right]^{G} .
$$

According to Lemma $2.3(a), \operatorname{Hyp}(\vartheta)^{G}$ is a multiplicative semigroup in the ring $\mathbb{C}\left[V^{\prime}\right]$. We will usually assume $\vartheta$ to be chosen in such a way that (2.9) contains some polynomials of positive degrees (not only constants). A few explicit examples of hyperbolic polynomials with a reflection group invariance are given below.

Example 2.4. The set $\operatorname{Hyp}(\vartheta, 0)$ consists of all non-vanishing constants, so that $\operatorname{Hyp}(\vartheta, 0)^{G} \equiv \operatorname{Hyp}(\vartheta, 0)$ holds trivially for any $G$ and any $\vartheta \in V^{\prime} \backslash\{0\}$.

Example 2.5. A linear form $v_{*}=(v, \cdot), v \in V$, belongs to $\operatorname{Hyp}(\vartheta, 1)$ if and only if $(v, \vartheta) \neq 0$. Hence, $\operatorname{Hyp}(\vartheta, 1)^{G}$ consists of all linear polynomials $v_{*}(\zeta) \in \mathbb{C}\left[V^{\prime}\right]$ such that $(v, \vartheta) \neq 0$ and $(v, \alpha)=0$ for all $\alpha \in \Re(G)$. Clearly, when $G$ is essential, i.e. $\operatorname{rk} G=n, \operatorname{Hyp}(\vartheta, 1)^{G}$ is empty.

Example 2.6. A quadratic polynomial $P \in \mathbb{C}\left[V^{\prime}\right]$ belongs to $\operatorname{Hyp}(\vartheta, 2)$ if and only if the quadric $P(\cdot) / P(\vartheta)$ has a Lorentzian signature $(-,+, \ldots,+, 0, \ldots, 0)$ and $\vartheta$ is a time-like vector relative to it. In particular, if $P \in \operatorname{Hyp}(\vartheta, 2)$ is complete, then it is strongly hyperbolic, $P \in \operatorname{Hyp}^{\circ}(\vartheta, 2)$, and may be reduced to the canonical form

$$
P(\zeta):=-\left(\zeta^{1}\right)^{2}+\left(\zeta^{2}\right)^{2}+\ldots+\left(\zeta^{n}\right)^{2}
$$


with $\vartheta:=( \pm 1,0, \ldots, 0)$ as a hyperbolicity direction. In that case, any finite reflection group $G_{0}$ of rank $\operatorname{rk} G_{0} \leq n-1$ preserving $\vartheta$ leaves (2.10) invariant. More generally, $P \in \operatorname{Hyp}(\vartheta, 2)^{G}$ if $G=G_{0} \times G_{1}$ with $G_{1} \cong \mathbb{Z}_{2}$ generated by reflection in $\vartheta^{\perp} \subset V$.

Example 2.7. Suppose that $\operatorname{rk} G \leq n-1$ and $\vartheta \in V^{\prime} \backslash\{0\}$ is $G$-invariant. Then $\operatorname{Hyp}(\vartheta)$ is an invariant subset in $\mathbb{C}\left[V^{\prime}\right]$. Indeed, if $P(\zeta) \in \operatorname{Hyp}(\vartheta)$, then

$$
P\left(g^{-1}(\xi+\vartheta t)\right)=P\left(g^{-1}(\xi)+\vartheta t\right) \neq 0,
$$

when $\operatorname{Im} t \neq 0$ and $\xi \in V^{\prime}$, since $g^{-1}(\xi) \in V^{\prime}$ is real (for all $g \in G$ ) and the hyperbolicity condition (2.1) holds.

Now we may construct $\operatorname{Hyp}(\vartheta)^{G} \subset \operatorname{Hyp}(\vartheta)$ using the properties of hyperbolic polynomials stated in Lemma 2.3 . First, we define a homomorphism of semigroups $\operatorname{Hyp}(\vartheta) \rightarrow \operatorname{Hyp}(\vartheta)^{G}, P \mapsto P^{G}$, by (multiplicative) averaging over $G$ :

$$
P^{G}(\zeta):=\prod_{g \in G} P(g(\zeta)) .
$$

Clearly, when $P \in \operatorname{Hyp}(\vartheta), P^{G}$ is $G$-invariant and $\vartheta$-hyperbolic (as a product of hyperbolic polynomials).

Second, we may polarize any $P \in \operatorname{Hyp}(\vartheta, p)^{G}$ by setting (see Lemma 2.3 $(c)$ )

$$
P(\zeta+\vartheta t)=\sum_{k=0}^{p} t^{k} P_{k}(\zeta) .
$$

Then, each polar $P_{k}(\cdot) \in \operatorname{Hyp}(\vartheta, p-k)^{G}$, since $\vartheta$ is a $G$-invariant vector. Further, if $P \in \operatorname{Hyp}(\vartheta)^{G}$ (take, e.g., (2.11) ) admits a polynomial factorization (2.3), then each $G$-invariant factor also belongs to $\operatorname{Hyp}(\vartheta)^{G}$.

Example 2.8. To give an illustration to the previous example, consider the symmetric group $G \cong A_{n-1}$ with a root system $\Re$ canonically realized in $V^{\prime} \cong \mathbb{R}^{n}$. Fix $\vartheta=(1,1, \ldots, 1)$ and let $P_{n}(\zeta):=\zeta^{1} \zeta^{2} \cdots \zeta^{n} \in \mathbb{R}[\zeta]$. Clearly, $P_{n}(\cdot) \in \operatorname{Hyp}(\vartheta, n)$. On the other hand, $\vartheta$ and $P_{n}$ are both invariant under all coordinate permutations, and hence $P_{n} \in \operatorname{Hyp}(\vartheta, n)^{G}$. The polarization (2.12) of $P_{n}$ along $\vartheta$ gives precisely the elementary symmetric functions $P_{k}(\zeta):=\sum \zeta^{1} \zeta^{2} \cdots \zeta^{k}$. Hence, $P_{k} \in \operatorname{Hyp}(\vartheta, k)^{G}$ for each $k=0,1, \ldots, n$. In particular, $P_{2}(\zeta):=\zeta^{1} \zeta^{2}+\zeta^{1} \zeta^{3}+\cdots$ is a second order wave polynomial which takes the canonical form (2.10) after a proper change of coordinates. In connection with the approximation theory of hyperbolic equations these symmetric polynomials were studied in [43] (see also [13]). Similar examples of 'elementary' invariant polynomials with the hyperbolicity property can be constructed for other classical root systems.

Example 2.9. Let $G$ be a finite reflection group on $V \cong V^{\prime}$ with a root system $\Re$. Fix a total ordering on $V$, and single out an open connected component $\Gamma_{+}$ in $V_{\text {reg }}:=V \backslash \bigcup \alpha^{\perp}, \alpha \in \Re$, consisting of positive vectors. Then $\Gamma_{+}$is a conical domain in $V$, referred to as a positive Weyl chamber. Consider

$$
P(\zeta):=\prod_{\alpha \in \Re}(\alpha, \zeta)
$$

a discriminant polynomial of the group $G$. Then $P(\cdot)$ is both $G$-invariant and hyperbolic with respect to any vector $\vartheta \in \Gamma_{+}$(so that $\Gamma_{+}$is a (global) hyperbolicity cone of $P$ ). Hence, $P \in \operatorname{Hyp}\left(\Gamma_{+}, p\right)^{G}$, where $p:=|\Re|$ is the total number of roots in the system $\Re$. 
The object of our study is a class of linear differential operators defined as an image of $\operatorname{Hyp}(\vartheta)^{G} \subset \mathbb{C}\left[V^{\prime}\right]$ under the composition of mappings

$$
\mathbb{C}\left[V^{\prime}\right] \stackrel{\nabla_{m}}{\longrightarrow} \mathcal{W}_{m} \stackrel{\text { Res }}{\longrightarrow} \mathfrak{D}^{G, m} .
$$

The notation used in (2.14) has been explained in Section $1: G$ is a finite reflection group of rank rk $G \leq n$ on $V, m \in M(G)$ is a multiplicity function on a root system $\Re(G)$ associated to $G, \nabla_{m}$ and Res are the Dunkl and Heckman maps related to the pair $(G, m)$. More precisely, we set

$$
\mathfrak{D}_{\mathrm{Hyp}}^{G, m}:=\left\{\mathcal{L} \in \mathfrak{D}^{G, m} \mid \mathcal{L}=\operatorname{Res} \nabla_{m} P(-i \zeta), P(\cdot) \in \operatorname{Hyp}(\vartheta)^{G}\right\} .
$$

By construction, the elements of $\mathfrak{D}_{\mathrm{Hyp}}^{G, m}$ are $G$-invariant linear partial differential operators with constant principal symbols $P \in \operatorname{Hyp}(\vartheta)^{G}$ and rational lower order coefficients with singularities located on reflection hyperplanes $\alpha^{\perp} \subset V$ of the group $G$.

Example 2.10. Choose $\vartheta \in V^{\prime}, \vartheta \neq 0$, to be a $G$-invariant vector in $V^{\prime}$, and let $P \in \operatorname{Hyp}(\vartheta, 2)^{G}$ be a complete second order wave polynomial. Then, it follows from (1.13) that

$$
\mathcal{L}:=\operatorname{Res} \nabla_{m} P(-i \zeta)=\square_{n}+\sum_{\alpha \in \Re_{+}} \frac{m_{\alpha}\left(m_{\alpha}+1\right)(\alpha, \alpha)}{(\alpha, x)^{2}}
$$

The operator (2.16) can be interpreted as a generalization of the classical EulerPoisson-Darboux (EPD) operator $(\operatorname{rk} G=1)$ to higher rank root systems ( $\operatorname{rk} G \leq$ $n-1)$.

The differential operators in $\mathfrak{D}_{\text {Hyp }}^{G, m}$ are not automatically hyperbolic, even though their principal symbols $P$ belong to $\operatorname{Hyp}(\vartheta)$. The latter is only known as a necessary condition of hyperbolicity for

$$
\mathcal{L}:=P(D)+\sum_{\nu<p} a_{\nu}(x) D^{\nu}, \quad D:=-i \frac{\partial}{\partial x}
$$

with (locally) smooth coefficients under lower order derivatives $a_{\nu}(\cdot) \in \mathcal{C}^{\infty}(\Omega)$, $\Omega \subset V$. In general, one has to impose additional restrictions (either on the singular points of the hyperbolic hypersurface $\Xi(P)$ or on the lower terms added) in order to guarantee the existence of a proper fundamental solution with conic support ('intrinsic' hyperbolicity). In fact, the operator (2.17) is hyperbolic for arbitrary lower order terms, only if $P \in \operatorname{Hyp}^{\circ}(\vartheta)$. For $P \in \operatorname{Hyp}(\vartheta)$ with multiple characteristics the situation is more delicate: addition of (even constant) lower terms may lead to the loss of hyperbolicity (see, e.g., [42, [54]). It goes beyond the purposes of the present paper to investigate precise conditions under which the operators in $\mathfrak{D}_{\mathrm{Hyp}}^{G, m}$ are hyperbolic in the case of arbitrary multiplicities $m \in M$. For our needs the following result (to be proved in the next section) is sufficient.

Theorem 2.11. Let $\Omega$ be an open connected set in $V_{\text {reg }}=V \backslash \bigcup \alpha^{\perp}, \alpha \in \Re$, and let $m \in M$ be a fixed multiplicity function on $\Re$. Denote by $\{m\} \in M$ the fractional part of $m$, so that $0 \leq\left\{m_{\alpha}\right\}<1$ for all $\alpha \in \Re$. Then, the operator $\mathcal{L}_{m}:=\operatorname{Res} \nabla_{m} P(-i \zeta) \in \mathfrak{D}_{\mathrm{Hyp}}^{G, m}$ is hyperbolic in $\Omega$ if and only if this is true for $\mathcal{L}_{\{m\}} \in \mathfrak{D}_{\mathrm{Hyp}}^{G,\{m\}}$. 
Corollary 2.12. Let $\mathfrak{m} \subset M$ be a lattice of integer-valued multiplicity functions in $M$. Then, for any fixed $m \in \mathfrak{m}$, the set $\mathfrak{D}_{\mathrm{Hyp}}^{G, m}$ is a multiplicative semigroup of hyperbolic operators isomorphic to $\operatorname{Hyp}(\vartheta)^{G}$.

2.3. Riesz kernels. Let $\mathcal{D}(\Omega) \subset \mathcal{C}^{\infty}(\Omega)$ be the space of all complex-valued $\mathcal{C}^{\infty}$ functions on $V$ with supports compactly embedded in an open set $\Omega \subseteq V$, and let $\mathcal{D}^{\prime}(\Omega)$ be the corresponding space of distributions. When $\Omega=V$, we usually omit the parentheses in this notation.

Suppose that $\Omega \subset V_{\text {reg }}$ is an open connected subset in $V_{\text {reg. Then, using the }}$ identification (1.12), we may (and will) regard $\mathcal{D}^{\prime}(\Omega)$ as a left unitary $\mathfrak{D}$-module on $V_{\text {reg }}^{\mathbb{C}}$.

Definition 2.13. The Riesz kernel of a regular differential operator $\mathcal{L} \in \mathfrak{D}$ with a constant hyperbolic principal symbol $P \in \operatorname{Hyp}(\vartheta)$ is a holomorphic (entire analytic) mapping $\Phi^{ \pm}(\mathcal{L}): \mathbb{C} \rightarrow \mathcal{D}^{\prime}(\Omega)$ with values in the space of distributions on $\Omega \subset V_{\text {reg }}$, such that

(i) $\quad \operatorname{supp} \Phi_{\lambda}^{ \pm}\left(\mathcal{L}, x, x_{0}\right) \subseteq \pm K\left(P, \vartheta, x_{0}\right)$,

(ii) $\quad \mathcal{L}\left[\Phi_{\lambda}^{ \pm}\left(\mathcal{L}, \cdot, x_{0}\right)\right](x)=\Phi_{\lambda-1}^{ \pm}\left(\mathcal{L}, x, x_{0}\right)$,

(iii) $\Phi_{0}^{ \pm}\left(\mathcal{L}, x, x_{0}\right)=\delta\left(x-x_{0}\right)$,

for any fixed $x_{0} \in \Omega$.

The operators $\mathcal{L}$ for which the kernel $\Phi_{\lambda}^{ \pm}(\mathcal{L})$ exists and is uniquely determined (up to inessential factors depending only on $\lambda$ ) will be called properly hyperbolic. We will write $\mathfrak{D}_{\text {Hyp }} \subset \mathfrak{D}$ for the class of such operators.

The value of a Riesz kernel $\lambda \mapsto \Phi_{\lambda}^{ \pm}\left(\mathcal{L}, x, x_{0}\right)$ at $\lambda=1$ is a fundamental solution $\Phi^{ \pm}\left(\mathcal{L}, x, x_{0}\right)$ of the differential operator $\mathcal{L}$ with support in $\pm K\left(P, \vartheta, x_{0}\right)$. Such a solution is clearly unique, and, following [57, we call it the principal fundamental solution of $\mathcal{L}$.

The classical example of a Riesz kernel [26] is the following family of homogeneous distributions $\Phi^{ \pm}(P): \mathbb{C} \rightarrow \mathcal{D}^{\prime}$ associated to a hyperbolic polynomial $P \in \operatorname{Hyp}(\vartheta)$ :

$$
\lambda \mapsto \Phi_{\lambda}^{ \pm}(P, x):=(2 \pi)^{-n} \int_{V^{\prime}} P(\zeta)^{-\lambda} e^{i(x, \zeta)} d \xi,
$$

where $\zeta:=\xi+i \eta \in T_{ \pm}(P, \vartheta)$ and $T_{ \pm}(P, \vartheta):=V^{\prime} \mp i \Gamma(P, \vartheta)$ is a tube domain in $V_{\mathbb{C}}^{\prime}$ spanned over the (global) hyperbolicity cone of $P$. Since $|P(\zeta)|$ has no zeros and $\arg P(\zeta)$ is continuous and single-valued on $T_{ \pm}(P, \vartheta)$ (once $\arg P( \pm \vartheta)$ is fixed), the definition of the complex power $P(\zeta)^{-\lambda}$ offers no difficulties, and the inverse Fourier-Laplace integral in (2.19) is absolutely convergent (in the distribution sense) for all $\lambda \in \mathbb{C}$. By Cauchy's theorem, this integral is independent of $\operatorname{Im} \zeta \in \pm \Gamma(P, \vartheta)$. In fact, we have

$$
\Phi_{\lambda}^{ \pm}(P, x)=\mathcal{F}^{-1}\left[P_{ \pm}(\xi)^{-\lambda}\right]
$$

where $\mathcal{F}^{-1}$ stands for the inverse Fourier transform on $V$ and $P_{ \pm}(\xi)^{\lambda} \in \mathcal{S}^{\prime}$ is definied as a temperate distribution on $V^{\prime}$ by the (weak) limit

$$
P_{ \pm}(\xi)^{\lambda}:=\lim _{\epsilon \rightarrow+0} P(\xi \mp i \epsilon \eta)^{\lambda}, \quad \eta \in \Gamma(P, \vartheta), \lambda \in \mathbb{C} .
$$

For later convenience, we summarize the basic analytic properties of distributions $\Phi_{\lambda}^{ \pm}(P, \cdot)$ in the following lemma. 
Lemma 2.14 (cf. 2], 26]). Let $P \in \operatorname{Hyp}(\vartheta, p)$. Then, the mapping $\Phi^{ \pm}(P): \mathbb{C} \rightarrow$ $\mathcal{D}^{\prime}, \lambda \mapsto \Phi_{\lambda}^{ \pm}(P, x)$, is entire analytic and the following properties hold:

$$
\begin{gathered}
\Phi_{\lambda}^{ \pm}(P, \kappa x)=\kappa^{\lambda p-n} \Phi_{\lambda}^{ \pm}(P, x), \quad \kappa>0, \\
\operatorname{supp} \Phi_{\lambda}^{ \pm}(P, x) \subseteq \pm K(P, \vartheta), \\
\operatorname{sing} \operatorname{supp} \Phi_{\lambda}^{ \pm}(P, x) \subseteq \pm W(P, \vartheta), \\
P(D) \Phi_{\lambda}^{ \pm}(P, x)=\Phi_{\lambda-1}^{ \pm}(P, x), \\
\Phi_{0}^{ \pm}(P, x)=\delta(x) .
\end{gathered}
$$

The formulas (2.22), (2.25), (2.26) follow immediately from (2.19); (2.23) is a direct consequence of the Paley-Wiener-Schwartz theorem applied to (2.20). The inclusion (2.24) means that the Riesz kernel distribution can be restricted to a $\mathcal{C}^{\infty}$-function on any open connected subset in $V$ outside the wave front surface $\pm W(P, \vartheta)$. In fact, as we will see below, $\Phi_{\lambda}^{ \pm}(P, x)$ is even locally holomorphic in $x$ everywhere in $V \backslash \pm W(P, \vartheta)$.

The purpose of this section is to construct an explicit representation for the Riesz kernel of a properly hyperbolic differential operator $\mathcal{L}$ in $\mathfrak{D}_{\text {Hyp }}^{G, m}$. This will be done in terms of distributions $\Phi_{\lambda}^{ \pm}(P, \cdot)$ associated with the hyperbolic polynomial $P \in \operatorname{Hyp}(\vartheta)^{G}$, the principal symbol of $\mathcal{L}$.

First, we introduce some convenient notation. Let ad $: \mathfrak{D} \times \mathfrak{D} \rightarrow \operatorname{End}_{\mathbb{C}}(\mathfrak{D})$ denote a bilinear map which associates to a pair of operators $\mathcal{L}, \mathcal{L}_{0} \in \mathfrak{D}$ a $\mathbb{C}$-linear endomorphism on the space $\mathfrak{D}$ :

$$
\operatorname{ad}\left(\mathcal{L}, \mathcal{L}_{0}\right): \mathfrak{D} \rightarrow \mathfrak{D}, \quad \Theta \mapsto \operatorname{ad}\left(\mathcal{L}, \mathcal{L}_{0}\right)[\Theta],
$$

such that

$$
\operatorname{ad}\left(\mathcal{L}, \mathcal{L}_{0}\right)[\Theta]:=\mathcal{L} \Theta-\Theta \mathcal{L}_{0}
$$

Given $N \in \mathbb{Z}_{+}$, we write $\operatorname{ad}^{N}\left(\mathcal{L}, \mathcal{L}_{0}\right): \mathfrak{D} \rightarrow \mathfrak{D}$ for the $N$-th iteration of (2.27):

$$
\operatorname{ad}^{N}\left(\mathcal{L}, \mathcal{L}_{0}\right):=\operatorname{ad}\left(\mathcal{L}, \mathcal{L}_{0}\right) \circ \operatorname{ad}^{N-1}\left(\mathcal{L}, \mathcal{L}_{0}\right)
$$

with the additional convention $\operatorname{ad}^{0}\left(\mathcal{L}, \mathcal{L}_{0}\right) \equiv \mathrm{id}$.

Lemma 2.15. Let $\mathcal{L}_{0}, \mathcal{L} \subset \mathfrak{D}$ be linear differential operators defined in a domain $\Omega \subset V_{\text {reg }}$, and let $\mathcal{L}_{0} \subset \mathfrak{D}_{\text {Hyp }}$ be properly hyperbolic. Suppose that there exist a regular function $\Theta(x) \in \mathcal{O}$, regarded as a (multiplication) operator in $\mathfrak{D}$, and a non-negative integer $N \in \mathbb{Z}_{+}$, such that $\Theta(x) \neq 0$ on $\Omega$ and

$$
\operatorname{ad}^{N+1}\left(\mathcal{L}, \mathcal{L}_{0}\right)[\Theta(x)]=0 \quad \text { identically in } \mathfrak{D} .
$$

Then, $\mathcal{L}$ is also a properly hyperbolic operator, $\mathcal{L} \subset \mathfrak{D}_{\mathrm{Hyp}}$, with the same principal symbol as $\mathcal{L}_{0}$, and the following relation between the Riesz kernels of $\mathcal{L}$ and $\mathcal{L}_{0}$ holds:

$$
\Phi_{\lambda}^{ \pm}\left(\mathcal{L}, x, x_{0}\right)=\Theta\left(x_{0}\right)^{-1} \sum_{k=0}^{N}(-1)^{k} \frac{(\lambda)_{k}}{k !} \operatorname{ad}^{k}\left(\mathcal{L}, \mathcal{L}_{0}\right)[\Theta(x)] \Phi_{\lambda+k}^{ \pm}\left(\mathcal{L}_{0}, x, x_{0}\right),
$$

where $(\lambda)_{k}:=\Gamma(\lambda+k) / \Gamma(\lambda), \lambda \in \mathbb{C}$. 
Proof. Let $z \in \mathbb{C}$ be an auxiliary complex variable. Define

$$
S_{x, z}:=\sum_{k=0}^{N} \frac{(-1)^{k}}{k !} \operatorname{ad}^{k}\left(\mathcal{L}, \mathcal{L}_{0}\right)[\Theta(x)] \frac{\partial^{k}}{\partial z^{k}}
$$

as a differential operator on $\mathcal{D}^{\prime}(\Omega) \otimes \mathbb{C}[[z]]$. Then, equation (2.30) is equivalent to the operator identity

$$
(\mathcal{L}-z) S_{x, z}=S_{x, z}\left(\mathcal{L}_{0}-z\right) .
$$

Indeed, in view of definitions (2.28), (2.29) and (2.32), we have

$$
(\mathcal{L}-z) S_{x, z}-S_{x, z}\left(\mathcal{L}_{0}-z\right)=\frac{(-1)^{N}}{N !} \operatorname{ad}^{N+1}\left(\mathcal{L}, \mathcal{L}_{0}\right)[\Theta(x)] \frac{\partial^{N}}{\partial z^{N}} .
$$

Further, if $\lambda \mapsto \Phi_{\lambda}^{ \pm}\left(\mathcal{L}_{0}, x, x_{0}\right)$ is a Riesz kernel of $\mathcal{L}_{0} \subset \mathfrak{D}_{\mathrm{Hyp}}$, and $x_{0} \in \Omega$ is fixed, we construct a map

$$
\Phi^{ \pm}\left(\mathcal{L}_{0}, x, x_{0} ; z\right): \mathbb{C} \rightarrow \mathcal{D}^{\prime}(\Omega) \otimes \mathbb{C}[[z]]
$$

letting

$$
\lambda \mapsto \Phi_{\lambda}^{ \pm}\left(\mathcal{L}_{0}, x, x_{0} ; z\right):=\sum_{k=0}^{\infty} \frac{(\lambda)_{k}}{k !} \Phi_{\lambda+k}^{ \pm}\left(\mathcal{L}_{0}, x, x_{0}\right) z^{k} .
$$

Clearly, (2.34) is (termwise) holomorphic in $\lambda$, and the following properties hold:

$$
\begin{gathered}
\left(\mathcal{L}_{0}-z\right)\left[\Phi_{\lambda}^{ \pm}\left(\mathcal{L}_{0}, \cdot, x_{0} ; z\right)\right](x)=\Phi_{\lambda-1}^{ \pm}\left(\mathcal{L}_{0}, x, x_{0} ; z\right), \\
\lim _{\lambda \rightarrow 0} \Phi_{\lambda}^{ \pm}\left(\mathcal{L}_{0}, x, x_{0} ; z\right)=\delta\left(x-x_{0}\right),
\end{gathered}
$$

in virtue of (2.18), (2.34). Note that evaluation of $\Phi_{\lambda}^{ \pm}\left(\mathcal{L}_{0}, x, x_{0} ; z\right)$ at $z=0$ gives precisely the Riesz kernel $\Phi_{\lambda}^{ \pm}\left(\mathcal{L}_{0}, x, x_{0}\right)$.

Now, setting

$$
\Phi_{\lambda}^{ \pm}\left(\mathcal{L}_{0}, x, x_{0} ; z\right):=\Theta\left(x_{0}\right)^{-1} S_{x, z}\left[\Phi_{\lambda}^{ \pm}\left(\mathcal{L}_{0}, \cdot, x_{0}, \cdot\right)\right](x, z),
$$

we observe that (2.35), (2.36) are valid also for (2.37), when $\mathcal{L}_{0}$ is replaced by $\mathcal{L}$. Indeed, the first equation

$$
(\mathcal{L}-z)\left[\Phi_{\lambda}^{ \pm}\left(\mathcal{L}, \cdot, x_{0} ; z\right)\right](x)=\Phi_{\lambda-1}^{ \pm}\left(\mathcal{L}, x, x_{0} ; z\right)
$$

follows from (2.35), (2.33) and the fact that $S_{x, z}$ does not depend explicitly on $\lambda$, while the second condition

$$
\lim _{\lambda \rightarrow 0} \Phi_{\lambda}^{ \pm}\left(\mathcal{L}, x, x_{0} ; z\right)=\delta\left(x-x_{0}\right)
$$

is a consequence of (2.36) and the inner structure of the operator $S_{x, z}$ (the $\partial_{z}$-free term of $S_{x, z}$ is a multiplication by the non-vanishing function $\Theta(x)$ and, hence, a globally invertible operator on $\mathcal{D}^{\prime}(\Omega)$ ).

It follows from $(2.38)$ and $(2.39)$ that

$$
\Phi_{\lambda}^{ \pm}\left(\mathcal{L}, x, x_{0}\right):=\Phi_{\lambda}^{ \pm}\left(\mathcal{L}, x, x_{0} ; 0\right)
$$

satisfies axioms (ii), (iii) of definition (2.18). On the other hand, the explicit formula (2.31) derived upon substitution of (2.32), (2.34) into (2.37) and (2.40) shows that condition $(i)$ holds as well. Hence, $\Phi_{\lambda}^{ \pm}\left(\mathcal{L}, x, x_{0}\right)$ is a Riesz kernel of the operator $\mathcal{L}$, and $\mathcal{L}$ is properly hyperbolic. The lemma is proven. 
Lemma 2.16. Let $m \in M$, and let $\{m\} \in M$ be the fractional part of $m, 0 \leq$ $\left\{m_{\alpha}\right\}<1, \alpha \in \Re$. Then, there exist a non-negative integer $N_{m} \in \mathbb{Z}_{+}$and a (polynomial) function $\Theta_{m} \in \mathbb{C}[V]$, having no zeros in $V_{\text {reg }}=V \backslash \bigcup \alpha^{\perp}$, such that

$$
\operatorname{ad}^{N_{m}+1}\left(\mathcal{L}_{m}, \mathcal{L}_{\{m\}}\right)\left[\Theta_{m}(x)\right]=0
$$

for any $\mathcal{L}_{m} \in \mathfrak{D}^{G, m}$ and $\mathcal{L}_{\{m\}} \in \mathfrak{D}^{G,\{m\}}$ corresponding to the same homogeneous polynomial $P \in \mathbb{C}\left[V^{\prime}\right]^{G}$.

Proof. Consider first the case of non-negative multiplicities $m_{\alpha} \geq 0, \forall \alpha \in \Re$.

Let $\varepsilon \in \operatorname{Char}(G)$ be an arbitrary character of $G$, and let $\theta_{\varepsilon} \in \mathbb{C}[V]_{\varepsilon}^{G}$ be the corresponding fundamental semi-invariant defined by (1.11). Regarding $\theta_{\varepsilon}$ as a multiplication operator on $\mathcal{O}$, we have (by Lemma 1.5.

$$
\operatorname{ad}_{\nabla_{m}(P)}^{N_{\varepsilon}+1}\left[\theta_{\varepsilon}\right]=0 \quad \text { for any } P \in \mathbb{C}\left[V^{\prime}\right]^{G} .
$$

It is easy to see, in fact, that $N_{\varepsilon}=\left|\mathcal{E}_{+}\right|:=\sum_{\alpha \in \Re_{+}} 1_{\varepsilon}(\alpha)$.

Since $\theta_{\varepsilon}$ maps $\mathcal{O}^{G}$ to $\mathcal{O}_{\varepsilon}^{G}$, the restriction Res applied to both sides of (2.42) gives (see Lemma 1.6)

$$
\operatorname{Res}\left(\operatorname{ad}_{\nabla_{m}(P)}^{N_{\varepsilon}+1}\left[\theta_{\varepsilon}\right]\right)=\operatorname{ad}^{N_{\varepsilon}+1}\left(\mathcal{L}_{m-1_{\varepsilon}}, \mathcal{L}_{m}\right)\left[\theta_{\varepsilon}\right]=0,
$$

where $\mathcal{L}_{m}:=\operatorname{Res} \nabla_{m}(P)$ and $\mathcal{L}_{m-1_{\varepsilon}}:=\operatorname{Res}^{\varepsilon} \nabla_{m}(P)$. To justify the latter notation, we observe that

$$
\operatorname{Res}^{\varepsilon} \nabla_{m}(P)=\operatorname{Res} \nabla_{m-1_{\varepsilon}}(P), \quad P \in \mathbb{C}\left[V^{\prime}\right]^{G} .
$$

Indeed, in view of formula (1.9) and Lemma [1.6] we have

$$
\operatorname{Res}^{\varepsilon} \nabla_{m}(P)=\sum_{k \geq 0} \frac{1}{2^{k} k !} \operatorname{Res}^{\varepsilon} \operatorname{ad}_{\Delta_{m}}^{k}\left[P_{k}\right]=\sum_{k \geq 0} \operatorname{ad}_{\operatorname{Res}^{\varepsilon} \Delta_{m}}^{k}\left[P_{k}\right],
$$

where $P_{k} \in \mathbb{C}_{k}[V]^{G}$, while $\operatorname{Res}^{\varepsilon} \Delta_{m}$ satisfies (2.44) by a straightforward calculation.

When $P \in \mathbb{C}\left[V^{\prime}\right]^{G}$ is homogeneous, $\mathcal{L}_{m}$ is formally self-adjoint (up to a sign depending only on the degree of $P$; see again (1.9)). Hence, we may interchange $\mathcal{L}_{m}$ and $\mathcal{L}_{m-1_{\varepsilon}}$ in (2.43):

$$
\operatorname{ad}^{N_{\varepsilon}+1}\left(\mathcal{L}_{m}, \mathcal{L}_{m-1_{\varepsilon}}\right)\left[\theta_{\varepsilon}(x)\right]=0 .
$$

Given a non-negative $m \in M$, we choose a finite set $I_{m} \subset \operatorname{Char}(G)$ of $G$ characters in such a way that

$$
m=\{m\}+\sum_{\varepsilon \in I_{m}} 1_{\varepsilon} .
$$

Then, letting $N_{m}:=\sum 1_{\varepsilon}$ and $\Theta_{m}:=C \prod \theta_{\varepsilon}, C \in \mathbb{C} \backslash\{0\}$, with summation and product over $I_{m}$, one can verify the identity (2.41), lowering the values $m_{\alpha}$, step by step, with the help of (2.46).

When $m_{\alpha}<0$ for some $\alpha \in \Re$, we may assume $m_{\alpha}<-1$ in view of (2.46). This case reduces, in turn, to the case of positive $m_{\alpha}$, since each $\mathcal{L}_{m} \in \mathfrak{D}^{G, m}$ depends on the values of $m$ only as $m_{\alpha}\left(m_{\alpha}+1\right)$ (cf. (1.9), (1.13) ), and, hence, it is invariant under the involution $m_{\alpha} \mapsto-m_{\alpha}-1$. The lemma is proven.

The results of Lemma 2.15] and Lemma 2.16 imply the statement of Theorem 2.11 In the sequel, with a view of studying lacunas, we will deal mainly with differential operators $\mathcal{L}_{m} \in \mathfrak{D}_{\text {Hyp }}^{G, m}$ parameterized by integer multiplicities. By 
Corollary 2.12, they are all properly hyperbolic. In fact, we will restrict our consideration to the case of non-negative $m_{\alpha} \in \mathbb{Z}_{+}$. Since $\mathfrak{D}^{G, m}=\mathfrak{D}^{G,-m-1}$, this does not lead to any loss of generality.

Thus, we conclude this section with the following theorem.

Theorem 2.17. Let $\mathfrak{m} \subset M$ be a lattice of integer-valued multiplicity functions in $M$, and let $\mathfrak{m}_{+}:=\left\{m \in M \mid m_{\alpha} \in \mathbb{Z}_{+}\right.$for all $\left.\alpha \in \Re\right\}$ denote its positive part. Then, each $\mathcal{L}_{m} \in \mathfrak{D}_{\mathrm{Hyp}}^{G, m}, m \in \mathfrak{m}_{+}$, is properly hyperbolic in any open connected subset $\Omega \subset V_{\text {reg }}$. The Riesz kernel of $\mathcal{L}_{m}$ and its principal fundamental solution are given respectively by the formulas

$$
\begin{gathered}
\Phi_{\lambda}^{ \pm}\left(\mathcal{L}_{m}\right)=\sum_{k=0}^{M}(-1)^{k} \frac{(\lambda)_{k}}{k !} \operatorname{ad}^{k}\left(\mathcal{L}_{m}, \mathcal{L}_{0}\right)\left[\Theta_{m}(x)\right] \Phi_{\lambda+k}^{ \pm}\left(P, x-x_{0}\right) \\
\Phi^{ \pm}\left(\mathcal{L}_{m}\right)=\sum_{k=0}^{M}(-1)^{k} \operatorname{ad}^{k}\left(\mathcal{L}_{m}, \mathcal{L}_{0}\right)\left[\Theta_{m}(x)\right] \Phi_{k+1}^{ \pm}\left(P, x-x_{0}\right)
\end{gathered}
$$

where $\Phi_{\lambda}^{ \pm}(P, \cdot)$ are distributions (2.19) associated to a hyperbolic polynomial $P \in$ $\operatorname{Hyp}(\vartheta)^{G}$, the principal symbol $\mathcal{L}_{m}$, and $\Theta_{m}(x):=\theta_{m}(x) / \theta_{m}\left(x_{0}\right)$ with

$$
\theta_{m}(x):=\prod_{\alpha \in \Re_{+}}(\alpha, x)^{m_{\alpha}}
$$

and

$$
M:=\operatorname{deg} \theta_{m}=\sum_{\alpha \in \Re_{+}} m_{\alpha} .
$$

Proof. The proof follows from Lemma 2.15 and Lemma 2.16.

\section{Regular lacunas and Petrovsky's Condition}

Let $\mathcal{L}$ be a properly hyperbolic differential operator with a constant principal symbol $P \in \operatorname{Hyp}(\vartheta)$, and let $\lambda \mapsto \Phi_{\lambda}^{ \pm}\left(\mathcal{L}, x, x_{0}\right)$ be a Riesz kernel associated with $\mathcal{L}$. We assume that $\mathcal{L}$ is defined in some open connected part $\Omega$ of $V \cong \mathbb{R}^{n}$ and has $\mathcal{C}^{\infty}$-smooth (or locally analytic) coefficients thereon.

Definition 3.1. Let $x_{0} \in \Omega$ be fixed. An open connected set $L^{ \pm}\left(x_{0}\right)$ of $V \backslash \pm W\left(P, \vartheta, x_{0}\right)$ is called a regular lacuna of the Riesz kernel $\Phi_{\lambda}^{ \pm}\left(\mathcal{L}, \cdot, x_{0}\right)$ at a point $\lambda$, if $L^{ \pm}\left(x_{0}\right) \subset \Omega$ and $L^{ \pm}\left(x_{0}\right) \cap \operatorname{supp} \Phi_{\lambda}^{ \pm}\left(\mathcal{L}, \cdot, x_{0}\right)$ is empty.

Generically, the support $\operatorname{supp} \Phi_{\lambda}^{ \pm}\left(\mathcal{L}, x, x_{0}\right)$ entirely fills the interior $\pm K^{\circ}\left(P, \vartheta, x_{0}\right)$ of the propagation cone for almost all $\lambda \in \mathbb{C}$. In this case the only regular lacuna of $\Phi_{\lambda}^{ \pm}$lies in the complement $V \backslash \pm K\left(P, \vartheta, x_{0}\right)$, and it is referred to as the trivial lacuna. The values of $\lambda \in \mathbb{C}$ for which $\Phi_{\lambda}^{ \pm}\left(\mathcal{L}, x, x_{0}\right)$ has nontrivial lacunas are called singular. For example, singular for any $\mathcal{L}$ are $\lambda=0,-1,-2, \ldots$, since $\Phi_{-k}^{ \pm}\left(\mathcal{L}, x, x_{0}\right)=$ $\mathcal{L}^{k}\left[\delta\left(x-x_{0}\right)\right]$, when $k \in \mathbb{Z}_{+}$, as follows from (2.18).

The main concern in the classical theory of lacunas ([50, [2], 33]) is to study the support structure of the principal fundamental distribution $\Phi^{ \pm}(P, \cdot)$ associated to a hyperbolic polynomial $P \in \operatorname{Hyp}(\vartheta)$. Thanks to Petrovsky's work, the basic idea in the theory is to represent the function $\Phi^{ \pm}(P, \cdot)$ locally (outside the wave front surface $\pm W(P, \vartheta)$ ) as an Abelian integral of a rational form (with poles at complex zeros of $P$ ) over properly constructed cycles in the complex projective 
space $\mathbb{C P}^{n-1}$. The vanishing of the cycle relative to a point $x \in \pm K^{\circ}(P, \vartheta)$ will then imply the vanishing of $\Phi^{ \pm}(P, x)$ in the vicinity of $x$ and, by analyticity, also inside the whole component $L^{ \pm} \subset \pm K(P, \vartheta) \backslash \pm W(P, \vartheta)$ containing this point. This provides an effective link between the theory of lacunas and the topology of projective algebraic surfaces in $\mathbb{C P}^{n-1}$.

In this section we employ a similar idea to study a lacunary structure of fundamental solutions for hyperbolic operators $\mathcal{L}$ in $\mathfrak{D}_{\mathrm{Hyp}}^{G, m}$. It appears to be possible due to formulas (2.48) and (2.49). Note that (2.48) (in conjunction with Lemma 2.14) implies

$$
\operatorname{sing} \operatorname{supp} \Phi_{\lambda}^{ \pm}\left(\mathcal{L}, \cdot, x_{0}\right) \subseteq W\left(P, \vartheta, x_{0}\right)
$$

for any $\mathcal{L}_{m} \in \mathfrak{D}_{\mathrm{Hyp}}^{G, m}, m \in \mathfrak{m}_{+}$, and $\lambda \in \mathbb{C}$. This condition is natural and, in fact, it is valid for a much wider class of hyperbolic operators. What is more specific is that the kernel $\Phi_{\lambda}^{ \pm}\left(\mathcal{L}, \cdot, x_{0}\right)$ may admit non-trivial lacunas 6 when $\operatorname{Re} \lambda>0$. Here, we focus on a closer study of the principal fundamental solution $(\lambda=1)$. Our analysis rests heavily on the work of Atiyah, Bott and Gårding [2. The reader is referred to [2] for more analytical details.

Let $\stackrel{\circ}{V^{\prime}}:=V^{\prime} \backslash\{0\}$. Given a polynomial $P \in \operatorname{Hyp}(\vartheta, p)$ and a point $x \in V$, we define a family $\mathfrak{V}=\mathfrak{V}(x, P, \vartheta)$ of $\mathcal{C}^{\infty}$-smooth real vector fields $v: \stackrel{\circ}{V^{\prime}} \rightarrow V^{\prime}, \xi \mapsto$ $v(\xi)$, with the following characteristic properties. For each $\xi \in \stackrel{\circ}{V^{\prime}}:(i) v(\xi) \in$ $\Gamma_{\xi}(P, \vartheta) \cap X$, where $X$ stands for a real hyperplane in $V^{\prime}$ dual to the point $x \in V$; (ii) $v(\kappa \xi)=|\kappa| v(\xi), \kappa \in \stackrel{\circ}{\mathbb{R}} ; \quad($ iii $) P(\xi \pm i \epsilon v(\xi)) \neq 0$, when $0<\epsilon \leq 1$.

When $x \notin \pm W(P, \vartheta)$, the family $\mathfrak{V}(x, P, \vartheta)$ is not empty, and any two elements of it are homotopic, i.e. may be deformed one into another through a $\mathcal{C}^{\infty}$-mapping $[0,1] \rightarrow \mathfrak{V}$ within the family $\mathfrak{V}$. Indeed, in view of definition (2.8), $x \in W(P, \vartheta)$ if and only if $\left(x, \Gamma_{\xi}\right) \geq 0$ for at least one point $\xi \in \stackrel{\circ}{V^{\prime}}$. Hence, $x \notin \pm W(P, \vartheta)$ implies the existence of vectors $\eta_{\mp}$ in $\Gamma_{\xi}(P, \vartheta)$ such that $\left(\eta_{-}, x\right)<0$ while $\left(\eta_{+}, x\right)>0$, and then, by convexity, $\Gamma_{\xi}(P, \vartheta) \cap X$ is not empty for any $\xi \in \stackrel{\circ}{V}^{\prime}$. Further, the homogeneity property $(i i)$ is consistent with $(i)$, since the local cone $\Gamma_{\xi}(P, \vartheta)=$ $\Gamma\left(P_{\xi}, \vartheta\right)$ depends on the double ray $\stackrel{\circ}{\mathbb{R}} \xi$ only. The condition $(i i i)$ is achieved by taking the elements $v(\xi)$, satisfying $(i)$ and $(i i)$ with $|v(\xi)|$ small enough when $|\xi|=1$. The homotopy of $\mathfrak{V}$ follows essentially from the inner continuity of the mapping $(\xi, P) \mapsto \Gamma_{\xi}(P, \vartheta)$ (see 2, Lemma 6.7).

Now, when $x \notin \pm W(P, \vartheta)$, we can replace the constant vector field $\xi \mapsto \mp \operatorname{Im} \zeta$ in the Fourier-Laplace integral (2.19) by a smooth field $\xi \mapsto \mp v(\xi) \in \mathfrak{V}(x, P, \vartheta)$ homotopic to it, and then perform a radial integration with the use of the homogeneity $(i i)$ of $v(\xi)$. By Cauchy's theorem, this does not alter the integral, since the properties $(i)$ and $($ iii) guarantee that the exponential in (2.19) will stay bounded and $\xi \mp i v(\xi)$ will stay away from the complex zeros of $P$.

Assume first that $p \lambda-n \notin \mathbb{Z}_{+}$. Then the procedure outlined above gives

$$
\Phi_{\lambda}^{ \pm}(P, x)=(2 \pi)^{-n} \int_{\gamma(\xi)=1} P(\zeta)^{-\lambda} \chi_{p \lambda-n}(-i(x, \xi)+0) \omega(\zeta)
$$

${ }^{6}$ For example, this seems never to happen for the Riesz kernels 2.27 related to inhomogeneous polynomials $P \in \operatorname{hyp}(\vartheta)$ (cf. [2]-3]). 
where $x \notin \pm W(P, \vartheta), \zeta=\xi \mp i v(\xi)$ with $v(\xi) \in \mathfrak{V}(x, P, \vartheta)$, and the integration is understood in the distribution sense with the measure $\omega(\zeta)$ defined by the Kronecker $(n-1)$-form

$$
\omega(\zeta):=\sum_{k=1}^{n}(-1)^{k-1} \zeta^{k} d \zeta^{1} \wedge \cdots \wedge d \zeta^{k-1} \wedge d \zeta^{k+1} \wedge \cdots \wedge d \zeta^{n}
$$

The function $\gamma: \stackrel{\circ}{V^{\prime}} \rightarrow \mathbb{R}_{+}, \xi \mapsto \gamma(\xi)$, is any smooth real positive function on $\stackrel{\circ}{ }^{\prime}$ absolutely homogeneous of degree 1, e.g. $\gamma(\xi)=|\xi|$. The distribution $\chi_{s}(i t+0) \in$ $\mathcal{D}^{\prime}(\mathbb{R})$ is defined (for any $s \in \mathbb{C} \backslash \mathbb{Z}_{+}$) as a (weak) boundary limit of the complex function

$$
z \mapsto \chi_{s}(z):=\Gamma(-s) z^{s}, \quad s \notin \mathbb{Z}_{+},
$$

holomorphic in the region $\operatorname{Re} z>0$.

To provide analytic continuation of the integral in (3.2) for all $\lambda \in \mathbb{C}$, we notice that $\chi_{s}(z)$, as a function of $s$, has simple poles at $s=0,1,2, \ldots$ for any fixed $z \in \mathbb{C}$ with $\operatorname{Re} z>0$. More precisely, when $s \in \mathbb{Z}_{+}$, we have the Laurent expansion

$$
\chi_{s+t}(z)=\chi_{s}^{o}(z) t^{-1}+\chi_{s}(z)+\mathcal{O}(t), \quad t \rightarrow 0,
$$

where the constant term (denoted for convenience also by $\chi_{s}(z)$ ) and the residue $\chi_{s}^{o}(z)$ are given respectively by

$$
\begin{gathered}
\chi_{s}(z)=\frac{(-1)^{s+1}}{s !} z^{s}\left(\log z-\Gamma^{\prime}(1)-\sum_{k=1}^{s} \frac{1}{k}\right), \\
\chi_{s}^{o}(z)=\frac{(-1)^{s+1}}{s !} z^{s} .
\end{gathered}
$$

Now the required analytic continuation may be performed via the operation $\chi_{s} \rightarrow \frac{d}{d t}\left[t \chi_{s+t}\right]_{t=0}$. The result reads

$$
\begin{aligned}
\Phi_{\lambda}^{ \pm}(P, x) & =(2 \pi)^{-n} \int_{\gamma(\xi)=1} P(\zeta)^{-\lambda} \chi_{p \lambda-n}(-i(x, \xi)+0) \omega(\zeta) \\
& -\frac{(2 \pi)^{-n}}{p} \int_{\gamma(\xi)=1} P(\zeta)^{-\lambda} \log P(\zeta) \chi_{p \lambda-n}^{o}(-i(x, \xi)) \omega(\zeta) .
\end{aligned}
$$

Here, we put $\chi_{s}^{o}(z) \equiv 0$ when $s \notin \mathbb{Z}_{+}$, while $\chi_{s}$ is defined either by (3.6) or by (3.4) according to whether $s \in \mathbb{Z}_{+}$or not.

The second integral in (3.8) vanishes unless $p \lambda-n \in \mathbb{Z}_{+}$, and it is a polynomial in $x$ otherwise. As an immediate consequence of formula (3.8), we note that the function $(\lambda, x) \mapsto \Phi_{\lambda}^{ \pm}(P, x)$ is holomorphic in $\lambda$ for all $\lambda \in \mathbb{C}$ and locally holomorphic in $x$ when $x \notin \pm W(P, \vartheta)$ (cf. Lemma 2.14 and (2.24) ).

Suppose that $x \notin \mp K(P, \vartheta) \cup \pm W(P, \vartheta)$. Then $-x \notin \pm K(P, \vartheta)$ and, according to Lemma 2.14 and (2.23),$\Phi_{\lambda}^{ \pm}(P,-x) \equiv 0$. Setting

$$
\Phi_{\lambda}^{ \pm}(P, x)=\Phi_{\lambda}^{ \pm}(P, x)-e^{i \pi(p \lambda-n)} \Phi_{\lambda}^{ \pm}(P,-x),
$$


we can eliminate (for all $\lambda \in \mathbb{C}$ ) the 'logarithmic term' in (3.8):

$$
\Phi_{\lambda}^{ \pm}(P, x)=\frac{i}{(2 \pi)^{n-1}} \int_{\gamma(\xi)=1} P(\zeta)^{-\lambda} \varrho_{p \lambda-n}((x, \xi)) \omega(\zeta),
$$

where $\varrho_{s}(t) \in \mathcal{D}^{\prime}(\mathbb{R})$ is defined by

$$
2 \pi i \varrho_{s}(t):=\chi_{s}(-i t+0)-e^{i \pi s} \chi_{s}(i t+0), \quad s \in \mathbb{C} .
$$

For (2.49) one needs to evaluate the Riesz kernel $\Phi_{\lambda}^{ \pm}(P, x)$ at real integer points $\lambda \in \mathbb{Z}$. More explicit formulas for the distribution $\varrho_{s}(t)$ in that case are given by

$$
\begin{gathered}
\varrho_{s}(t)=\frac{1}{2} \frac{(i t)^{s}}{s !} \operatorname{sgn} t, \quad s=0,1,2, \ldots, \\
\varrho_{s}(t)=(-i)^{-s} \delta^{(-s-1)}(t), \quad s=-1,-2,-3, \ldots .
\end{gathered}
$$

In fact, the first formula (3.11) follows immediately from (3.6) and (3.10), while the second follows from the first, if we observe that $\varrho_{s}^{\prime}=i \varrho_{s-1}$ for all $s \in \mathbb{C}$.

Letting $\lambda=k, k \in \mathbb{Z}_{+} \backslash\{0\}$, in (3.9) and using (2.26), (2.27), we obtain the following formulas for the distribution $\Phi_{k}^{ \pm}(P, \cdot)$ localized in the vicinity of $x \notin$ $\mp K(P, \vartheta) \cup \pm W(P, \vartheta)$ :

$$
\Phi_{k}^{ \pm}(P, x)=\frac{i^{p k-n+1}}{2(2 \pi)^{n-1}(p k-n) !} \int_{\gamma(\xi)=1} P(\zeta)^{-k}(x, \xi)^{p k-n} \operatorname{sgn}(x, \xi) \omega(\zeta),
$$

when $p k \geq n$, and

$$
\Phi_{k}^{ \pm}(P, x)=-\frac{(-i)^{n-p k+1}}{(2 \pi)^{n-1}} \int_{\gamma(\xi)=1} P(\zeta)^{-k} \delta^{(n-p k-1)}((x, \xi)) \omega(\zeta),
$$

when $p k<n$.

The next step is to associate with the family $\mathfrak{V}(x, P, \vartheta)$ certain cycles and relative cycles in the complex projective space and to rewrite the right-hand sides of (3.13), (3.14) as rational integrals over them.

Let $P \in \operatorname{Hyp}(\vartheta), x \in V \backslash \pm W(P, \vartheta)$ and $v(\xi) \in \mathfrak{V}(x, P, \vartheta)$ be fixed as above. Consider the integration chain in (3.13), (3.14):

$$
\gamma^{\sim}:=\left\{\xi \in \stackrel{\circ}{V^{\prime}} \mid \gamma(\xi)=1\right\} \subset V^{\prime} .
$$

We assume $\gamma^{\sim}$ to be oriented in such a way that the $(n-1)$-form $(x, \xi) \omega(\xi)$ stays positive on $\gamma^{\sim}$, while the space $V^{\prime}$ is endowed with the standard orientation $d \xi>0$.

Following [2], we define the smooth 'complex shift' map

$$
\sigma_{ \pm}(x, v): \stackrel{\circ}{V^{\prime}} \rightarrow V_{\mathbb{C}}^{\prime}, \quad \xi \mapsto \xi \mp i v(\xi),
$$

and consider the image of the chain $\gamma^{\sim}$ in $V_{\mathbb{C}}^{\prime}$ under (3.16):

$$
\sigma_{ \pm}\left(x, v ; \gamma^{\sim}\right):=\operatorname{Im}_{\sigma_{ \pm}}\left[\gamma^{\sim}\right] .
$$

Let

$$
\Xi_{\mathbb{C}}:=\left\{\zeta \in V_{\mathbb{C}}^{\prime} \mid P(\zeta)=0\right\}, \quad X_{\mathbb{C}}:=\left\{\zeta \in V_{\mathbb{C}}^{\prime} \mid(x, \zeta)=0\right\}
$$

be the complex counterparts of the real surfaces $\Xi(P)$ and $X$. Then $\sigma_{ \pm}\left(x, v ; \gamma^{\sim}\right)$ is a relative cycle of the pair $\left(V_{\mathbb{C}}^{\prime} \backslash \Xi_{\mathbb{C}}, X_{\mathbb{C}} \backslash\left(X_{\mathbb{C}} \cap \Xi_{\mathbb{C}}\right)\right)$ oriented by $\omega(\zeta) \operatorname{sgn}(x, \operatorname{Re} \zeta)>0$. By construction, $\sigma_{ \pm}\left(x, v ; \gamma^{\sim}\right)$ is homologous to $\gamma^{\sim}$ in $V_{\mathbb{C}}^{\prime} \backslash \Xi_{\mathbb{C}}$ for any $v \in \mathfrak{V}(x, P, \vartheta)$. 
Since the mapping $\xi \mapsto v(\xi)$ is absolutely homogeneous (of degree 1), it is relevant to project the cycle (3.17) onto $\mathbb{C P}^{n-1}$ :

$$
\sigma_{ \pm}^{*}\left(x, v ; \gamma^{\sim}\right):=\operatorname{Im}_{\pi}\left[\sigma_{ \pm}\left(x, v ; \gamma^{\sim}\right)\right]
$$

via the canonical surjection $\pi: \mathbb{C}^{n} \backslash\{0\} \rightarrow \mathbb{C P}^{n-1}$.

Let $\Xi_{\mathbb{C}}^{*}$ and $X_{\mathbb{C}}^{*}$ stand for the projective images of $\Xi_{\mathbb{C}}$ and $X_{\mathbb{C}}$ in $\mathbb{C P}^{n-1}$. A representative of the relative homology class

$$
\left[\sigma_{ \pm}^{*}(x)\right] \in H_{n-1}\left(\mathbb{C P}^{n-1} \backslash \Xi_{\mathbb{C}}^{*}, X_{\mathbb{C}}^{*} \backslash\left(X_{\mathbb{C}}^{*} \cap \Xi_{\mathbb{C}}^{*}\right) ; \mathbb{C}\right)
$$

generated by (3.18) is called the relative Petrovsky cycle.

Since the family $\mathfrak{V}(x, P, \vartheta)$ is one homotopy class, $\left[\sigma_{ \pm}^{*}(x)\right]$ does not depend on the choice of $v \in \mathfrak{V}$. Moreover, the homology class $\left[\sigma_{ \pm}^{*}(x)\right]$ is also independent of $\gamma^{\sim}$ and locally independent of $x \in V \backslash \pm W(P, \vartheta)$. This justifies the notation (3.19).

Taking the boundary of $\left[\sigma_{ \pm}^{*}(x)\right]$,

$$
\partial\left[\sigma_{ \pm}^{*}(x)\right] \in H_{n-2}\left(X_{\mathbb{C}}^{*} \backslash\left(X_{\mathbb{C}}^{*} \cap \Xi_{\mathbb{C}}^{*}\right) ; \mathbb{C}\right),
$$

gives an (absolute) homology in $X_{\mathbb{C}}^{*} \backslash\left(X_{\mathbb{C}}^{*} \cap \Xi_{\mathbb{C}}^{*}\right)$. The absolute Petrovsky cycle $\beta_{ \pm}^{*}(x)$ is then defined as a representative of the class

$$
\left[\beta_{ \pm}^{*}(x)\right]:=\frac{1}{2} \mathfrak{t}_{x}^{*} \partial\left[\sigma_{ \pm}^{*}(x)\right] \in H_{n-1}\left(\mathbb{C P}^{n-1} \backslash\left(X_{\mathbb{C}}^{*} \cup \Xi_{\mathbb{C}}^{*}\right) ; \mathbb{C}\right)
$$

where $\mathfrak{t}_{x}^{*}$ denotes the map induced on homology by the Leray tube operation $\mathfrak{t}_{x}$ from $X_{\mathbb{C}}^{*} \backslash\left(X_{\mathbb{C}}^{*} \cap \Xi_{\mathbb{C}}^{*}\right)$ to $\mathbb{C P}^{n-1} \backslash\left(X_{\mathbb{C}}^{*} \cup \Xi_{\mathbb{C}}^{*}\right)$. The operation $\mathfrak{t}_{x}$ associates with each point $\zeta \in X_{\mathbb{C}}^{*} \backslash\left(X_{\mathbb{C}}^{*} \cap \Xi_{\mathbb{C}}^{*}\right)$ the boundary of a small neighborhood of this point in the real 2-plane in $\mathbb{C P}^{n-1}$ orthogonal to $X_{\mathbb{C}}^{*}$ at $\zeta$. More precisely, given a compact chain $\sigma \subset X_{\mathbb{C}} \backslash\left(X_{\mathbb{C}} \cap \Xi_{\mathbb{C}}\right), \mathfrak{t}_{x} \sigma$ can be viewed as a product $\{|(x, \zeta)|=\rho\} \times \sigma$ with $\rho$ so small that $\mathfrak{t}_{x} \sigma \subset \mathbb{C}^{n} \backslash \Xi_{\mathbb{C}}$. The orientation of $\mathfrak{t}_{x} \sigma$ is a product of the orientation of the complex plane with coordinate $(x, \zeta)$ and the orientation of $\sigma$.

Now let $p k \geq n$ and return to the first integral formula (3.13). Clearly,

$$
\int_{\gamma(\xi)=1} P(\zeta)^{-k}(x, \xi)^{p k-n} \operatorname{sgn}(x, \xi) \omega(\zeta)=\int_{\sigma_{ \pm}\left(x, v ; \gamma^{\sim}\right)} P(\zeta)^{-k}(x, \zeta)^{p k-n} \omega(\zeta)
$$

with $\zeta=\xi \mp i v(\xi)$ as an integration variable. The integrand in the right-hand side of (3.22) has the form $F(\zeta) \omega$, where $F(\zeta)$ is a rational function in $\zeta$ homogeneous of degree $-n$. Such a differential form is invariant under coordinate changes $\zeta_{j} \rightarrow$ $f(\zeta) \zeta_{j}$ and, hence, is a pull-back of a differential form on $\mathbb{C P}^{n-1}$ under the canonical projection $\pi: \mathbb{C}^{n} \backslash\{0\} \rightarrow \mathbb{C P}^{n-1}$.

Letting $\zeta=\left(\zeta_{1}: \ldots: \zeta_{n}\right)$ denote homogeneous coordinates in $\mathbb{C P}^{n-1}$, we get from (3.18) and (3.22)

$$
\Phi_{k}^{ \pm}(P, x)=\frac{\pi i^{p k-n+1}}{(2 \pi)^{n}(p k-n) !} \int_{\sigma_{ \pm}^{*}(x)} P(\zeta)^{-k}(x, \zeta)^{p k-n} \omega(\zeta),
$$

where $\sigma_{ \pm}^{*}(x)$ is a relative Petrovsky cycle corresponding to the point $x \notin$ $\mp K(P, \vartheta) \backslash \pm W(P, \vartheta)$, and $\omega(\zeta)$ is the Kronecker form on $\mathbb{C P}^{n-1}$ given (in terms of homogeneous coordinates) by (3.3). The integral (3.23) depends only on the homology class $\left[\sigma_{ \pm}^{*}(x)\right]$ of the cycle $\sigma_{ \pm}^{*}(x)$ in $H_{n-1}\left(\mathbb{C P}^{n-1} \backslash \Xi_{\mathbb{C}}^{*}, X_{\mathbb{C}}^{*} \backslash\left(X_{\mathbb{C}}^{*} \cap \Xi_{\mathbb{C}}^{*}\right) ; \mathbb{C}\right)$, since its integrand is a closed form of highest degree holomorphic on $\mathbb{C P} \mathbb{P}^{n-1} \backslash \Xi_{\mathbb{C}}^{*}$. 
Consider the opposite case $p k<n$. Formula (3.14) implies

$$
\Phi_{k}^{ \pm}(P, x)=-\frac{i^{n-p k+1}}{(2 \pi)^{n-1}} \int_{\frac{1}{2} \partial \sigma_{ \pm}\left(x, v ; \gamma^{\sim}\right)}(x, \partial / \partial \zeta)^{n-p k-1} P(\zeta)^{-k} \omega_{X}(\zeta),
$$

where the integration is taken over the $(n-2)$-cycle on $X_{\mathbb{C}} \backslash\left(X_{\mathbb{C}} \cap \Xi_{\mathbb{C}}\right)$

$$
\frac{1}{2} \partial \sigma_{ \pm}\left(x, v ; \gamma^{\sim}\right):=\left\{\zeta=\xi \mp i v(\xi) \mid \xi \in X \cap \gamma^{\sim}\right\}
$$

with a Leray measurd $7 \omega_{X}>0$ associated with the real hyperplane $X$ :

$$
\omega(\xi)=d(x, \xi) \wedge \omega_{X}(\xi)+\mathcal{O}((x, \xi)) .
$$

The Cauchy residue formula applied to (3.24) gives

$$
\Phi_{k}^{ \pm}(P, x)=-\frac{i^{n-p k}(n-p k-1) !}{(2 \pi)^{n}} \int P(\zeta)^{-k}(x, \zeta)^{p k-n} d(x, \zeta) \wedge \omega_{X}(\zeta)
$$

with integration over the product chain

$$
\beta_{ \pm}\left(x, v ; \gamma^{\sim}\right):=\frac{1}{2}\{|(x, \zeta)|=\rho\} \times \partial \sigma_{ \pm}\left(x, v ; \gamma^{\sim}\right), \quad \rho>0 \text { small enough }
$$

oriented by $\omega(\operatorname{Re}(x, \zeta), \operatorname{Im}(x, \zeta)) \wedge \omega_{X}(\zeta)>0$.

In view of homogeneity the integrand in (3.26) can be written in terms of homogeneous coordinates in $\mathbb{C P}^{n-1}$ (see the argument following equation (3.22)). Then the projective image $\beta_{ \pm}^{*}$ of (3.27) represents the homology class (3.21) and, hence, is an absolute Petrovsky cycle. As in the previous case, the result of integration will be independent of the choice of an individual representative in $\left[\beta_{ \pm}^{*}(x)\right]$ but determined by the class $\left[\beta_{ \pm}^{*}(x)\right]$ as a whole. Thus, we have

$$
\Phi_{k}^{ \pm}(P, x)=-\frac{i^{n-p k}(n-p k-1) !}{(2 \pi)^{n}} \int_{\beta_{ \pm}^{*}(x)} P(\zeta)^{-k}(x, \zeta)^{p k-n} \omega(\zeta),
$$

when $k \in \mathbb{Z}_{+} \backslash\{0\}$ and $p k<n$.

Equations (3.23) , (3.28) are essentially the classical Herglotz-Petrovsky-Leray formulas (cf. [2]). The following theorem gives a generalization of these formulas for hyperbolic operators from the class $\mathfrak{D}_{\mathrm{Hyp}}^{G, m}$.

Theorem 3.2. Let $\mathcal{L}_{m} \in \mathfrak{D}_{\text {Hyp }}^{G, m}, m \in \mathfrak{m}_{+}$, and let $P \in \operatorname{Hyp}(\vartheta, p)^{G}$ be the principal symbol of $\mathcal{L}_{m}$. Fix $x_{0} \in \Omega \subset V_{\text {reg }}$, and assume that $x \in \Omega$ and $x \notin \mp K\left(P, \vartheta, x_{0}\right) \cup$ $\pm W\left(P, \vartheta, x_{0}\right)$. Then, the principal fundamental solution $\Phi^{ \pm}\left(\mathcal{L}_{m}, x, x_{0}\right)$ is holomorphic in the vicinity of $x$ and is determined by the following formulas:

$$
\Phi^{ \pm}\left(x, x_{0}\right)=\sum_{k=1}^{M+1} C_{k} \operatorname{ad}^{k-1}\left(\mathcal{L}_{m}, \mathcal{L}_{0}\right)\left[\Theta_{m}\right] \int_{\bar{\beta}_{ \pm}^{*}} P(\zeta)^{-k}(\bar{x}, \zeta)^{p k-n} \omega(\zeta),
$$

\footnotetext{
${ }^{7}$ For the precise definition of Leray form associated with a regular hypersurface see, e.g., ref. 29], Ch.III.
} 
when $M<n / p-1$,

$$
\begin{aligned}
\Phi^{ \pm}\left(x, x_{0}\right) & =\sum_{k=1}^{\left[\frac{n-1}{p}\right]} C_{k} \operatorname{ad}^{k-1}\left(\mathcal{L}_{m}, \mathcal{L}_{0}\right)\left[\Theta_{m}\right] \int_{\bar{\beta}_{ \pm}^{*}} P(\zeta)^{-k}(\bar{x}, \zeta)^{p k-n} \omega(\zeta) \\
& +\sum_{k=\left[\frac{n-1}{p}\right]+1}^{M+1} \tilde{C}_{k} \operatorname{ad}_{k-1}\left(\mathcal{L}_{m}, \mathcal{L}_{0}\right)\left[\Theta_{m}\right] \int_{\bar{\sigma}_{ \pm}^{*}} P(\zeta)^{-k}(\bar{x}, \zeta)^{p k-n} \omega(\zeta)
\end{aligned}
$$

when $0<n / p-1 \leq M$, and

$$
\Phi^{ \pm}\left(x, x_{0}\right)=\sum_{k=1}^{M+1} \tilde{C}_{k} \operatorname{ad}^{k-1}\left(\mathcal{L}_{m}, \mathcal{L}_{0}\right)\left[\Theta_{m}\right] \int_{\bar{\sigma}_{ \pm}^{*}} P(\zeta)^{-k}(\bar{x}, \zeta)^{p k-n} \omega(\zeta),
$$

when $n \leq p$. Here, the integration is carried out over an absolute $\bar{\beta}_{ \pm}^{*}:=\beta_{ \pm}^{*}(\bar{x})$ and a relative $\bar{\sigma}_{ \pm}^{*}:=\sigma_{ \pm}^{*}(\bar{x})$ Petrovsky cycle associated with the point $\bar{x}=x-x_{0}$ (as defined in (3.19) and (3.21)); the constants $C_{k}$ and $\tilde{C}_{k}$ are given explicitly by

$$
C_{k}:=\frac{(-1)^{k} i^{n-p k}(n-p k-1) !}{(2 \pi)^{n}}, \quad \tilde{C}_{k}:=\frac{(-1)^{k-1} i^{p k-n+1} \pi}{(2 \pi)^{n}(p k-n) !},
$$

and $\Theta_{m} \in \mathfrak{D}$ is a multiplication operator by $\theta_{m}(x) / \theta_{m}\left(x_{0}\right)$ with the same $\theta_{m}(x)$ and $M$ as in Theorem 2.17 (see (2.50) and (2.51)).

Proof. Formulas (3.29)-(3.31) follow from Theorem 2.8 and (3.23), (3.28). By construction, all $\mathcal{L}_{m}$ have rational coefficients with poles located only on hyperplanes $\alpha^{\perp}, \alpha \in \Re$. Hence, so do the differential operators $\operatorname{ad}^{k}\left(\mathcal{L}_{m}, \mathcal{L}_{0}\right)\left[\theta_{m}(x)\right]$. This ensures analyticity of $\Phi^{ \pm}\left(\mathcal{L}_{m}, x, x_{0}\right)$, when $x$ stays away from movable $\left( \pm W\left(P, \vartheta, x_{0}\right)\right)$ and fixed $\left(\bigcup \alpha^{\perp}, \alpha \in \Re\right)$ singularity loci.

As in the classical case, the importance of the Herglotz-Petrovsky type formulas (3.29)-3.31) is that they provide sufficient conditions for existence of regular lacunas.

Corollary 3.3. Let $\mathcal{L}_{m} \in \mathfrak{D}_{\mathrm{Hyp}}^{G, m}, m \in \mathfrak{m}_{+}$, and let $P \in \operatorname{Hyp}(\vartheta, p)^{G}$, the principal symbol of $\mathcal{L}_{m}$, be a complete polynomial. Suppose that

$$
\sum_{\alpha \in \Re_{+}} m_{\alpha}<\frac{n}{p}-1
$$

and

$$
\partial\left[\sigma_{ \pm}^{*}(\bar{x})\right]=0 \quad \text { in } \quad H_{n-2}\left(\bar{X}_{\mathbb{C}}^{*} \backslash\left(\bar{X}_{\mathbb{C}}^{*} \cap \Xi_{\mathbb{C}}^{*}\right) ; \mathbb{C}\right)
$$

relative to some point $x \in \pm K\left(P, \vartheta, x_{0}\right) \backslash \pm W\left(P, \vartheta, x_{0}\right)$. Then, the open component $L^{ \pm}\left(x_{0}\right) \subset \pm K\left(P, \vartheta, x_{0}\right) \backslash \pm W\left(P, \vartheta, x_{0}\right)$ containing this point is a regular lacuna of the distribution $\Phi^{ \pm}\left(\mathcal{L}_{m}, x, x_{0}\right)$.

Proof. When $m \in \mathfrak{m}_{+}$satisfies condition (3.32), the fundamental solution of $\mathcal{L}_{m}$ is determined by (3.29). The class $\left[\sigma_{ \pm}^{*}(\bar{x})\right]$ is locally independent of $x$ (provided $x_{0}$ is fixed); hence the same is true for $\partial\left[\sigma_{ \pm}^{*}(\bar{x})\right]$ and $\left[\beta_{ \pm}^{*}(\bar{x})\right]$. On the other hand, if (3.33) holds, then $\left[\beta_{ \pm}^{*}(\bar{x})\right]=0$, and each term in the sum (3.29) vanishes in the vicinity of $x$. By analytical continuation, this must also be valid in the entire component $L^{ \pm}\left(x_{0}\right)$ outside the singularity loci. The proof is finished. 
Remark 3.4. Equation (3.33) is referred to as Petrovsky's condition in the form of Atiyah, Bott and Gårding. The stronger requirement

$$
\left[\sigma_{ \pm}^{*}(\bar{x})\right]=0 \quad \text { in } \quad H_{n-1}\left(\mathbb{C P}^{n-1} \backslash \Xi_{\mathbb{C}}^{*}, \bar{X}_{\mathbb{C}}^{*} \backslash\left(\bar{X}_{\mathbb{C}}^{*} \cap \Xi_{\mathbb{C}}^{*}\right) ; \mathbb{C}\right)
$$

connected with (3.30) and (3.31) would also imply that $x$ belonged to a regular lacuna for $\mathcal{L}_{m}$, even though (3.32) were not satisfied. This is indeed the case when $x \notin \pm K\left(P, \vartheta, x_{0}\right)$, since then we may choose $v(\xi)=\gamma(\xi) \eta \in \mathfrak{V}(\bar{x}, P, \vartheta)$ with $\eta \in$ $\Gamma(P, \vartheta) \cap \bar{X}$ in (3.18), so that the homotopy $\xi \mp i \gamma(\xi) \eta \rightarrow t \xi \mp i \gamma(\xi) \eta, t \in[0,1]$, contracts $\sigma_{ \pm}^{*}\left(\bar{x}, v ; \gamma^{\sim}\right)$ in $\mathbb{C P}^{n-1} \backslash \Xi_{\mathbb{C}}^{*}$ to the point $(\mp i \gamma(\xi) \eta)^{*}$ in $\bar{X}_{\mathbb{C}}^{*} \backslash\left(\bar{X}_{\mathbb{C}}^{*} \cap \Xi_{\mathbb{C}}^{*}\right)$. However, as shown in [3], the class $\left[\sigma_{ \pm}^{*}\left(x, v ; \gamma^{\sim}\right)\right]$ has a nonzero intersection number with $\left[\Gamma^{*}(P, \vartheta)\right] \in H_{n-1}\left(\mathbb{C P}^{n-1} \backslash \Xi_{\mathbb{C}}^{*}, \bar{X}_{\mathbb{C}}^{*} \backslash\left(\bar{X}_{\mathbb{C}}^{*} \cap \Xi_{\mathbb{C}}^{*}\right) ; \mathbb{C}\right)$ when $x$ is inside $\pm K\left(P, \vartheta, x_{0}\right)$ and, hence, cannot vanish in that case. Thus, (3.34) characterizes essentially the trivial lacunas.

Remark 3.5. If the Petrovsky condition (3.33) holds for some $P \in \operatorname{Hyp}(\vartheta, p)^{G}$ but inequality (3.32) does not, the corresponding component $L^{ \pm}\left(x_{0}\right)$ is a weak lacuna of $\Phi^{ \pm}\left(\mathcal{L}_{m}, \cdot, x_{0}\right)$. More precisely, when restricted to $L^{ \pm}\left(x_{0}\right), \Phi^{ \pm}\left(\mathcal{L}_{m}, x, x_{0}\right)$ is a rational function in $x$ and $x_{0}$ of homogeneity $p-n$,

$$
\Phi^{ \pm}\left(\mathcal{L}_{m}, \kappa x, \kappa x_{0}\right)=\kappa^{p-n} \Phi^{ \pm}\left(\mathcal{L}_{m}, x, x_{0}\right), \quad \kappa>0 .
$$

Indeed, if (3.32) is not valid, we have $p k-n \geq 0$ for some $k \in \mathbb{Z}_{+}, k \leq M+1$, and then $\Phi_{k}^{ \pm}(P, \bar{x})$ is a polynomial on $L^{ \pm}\left(x_{0}\right)$ of degree $p k-n$ homogeneous in $\bar{x}$. This follows from Herglotz-Petrovsky-Leray type formulas for higher derivatives of $\Phi_{k}^{ \pm}(P, \cdot)$,

$$
D^{\nu} \Phi_{k}^{ \pm}(P, \bar{x}) \sim \int_{\beta_{ \pm}^{*}(\bar{x})} P(\zeta)^{-k} \zeta^{\nu}(\bar{x}, \zeta)^{p k-|\nu|-n} \omega(\zeta), \quad|\nu|>p k-n,
$$

which can be derived along the same lines as (3.31). In view of Lemma 1.4 $(c)$ and definitions (2.15), (2.27), the operator $\theta_{m}\left(x_{0}\right)^{-1} \operatorname{ad}^{k-1}\left(\mathcal{L}_{m}, \mathcal{L}_{0}\right)\left[\theta_{m}(x)\right] \in \mathfrak{D}$ acting on $\Phi_{k}^{ \pm}(P, \bar{x})$ decreases its homogeneity by $p(k-1)$. As a result, each non-vanishing term in (3.30) or (3.31) is a rational function of degree $(p k-n)-p(k-1)=p-n$.

In general (cf. [2]), one can characterize a weak lacuna $L^{ \pm}\left(x_{0}\right)$ by the property that the fundamental distribution $\Phi^{ \pm}\left(\mathcal{L}, x, x_{0}\right)$ admits a $\mathcal{C}^{\infty}$-extension from $L^{ \pm}\left(x_{0}\right)$ to $\overline{L^{ \pm}\left(x_{0}\right)} \cap U\left(x_{0}\right)$ for some open neighborhood $U\left(x_{0}\right) \subset \Omega$ of the point $x_{0}$. With this definition, if $L^{ \pm}\left(x_{0}\right)$ is a regular weak lacuna of $\mathcal{L}_{m}$, then $\Phi^{ \pm}\left(\mathcal{L}_{m}, x, x_{0}\right)$ is a rational function of homogeneity $p-n$ therein. Indeed, it is immediate from (2.49) that any regular weak lacuna $L^{ \pm}\left(x_{0}\right)$ of $\mathcal{L}_{m}$ is a regular weak lacuna of its principal part $P(D)$. This implies that all derivatives $\left(\frac{\partial}{\partial t} x\right)^{\nu} \Phi_{k}^{ \pm}(P, t \bar{x})$ are bounded when $t \rightarrow 0$, provided $x$ is kept in $L^{ \pm}\left(x_{0}\right)$. In view of homogeneity (Lemma 2.14, (2.22)), we have in that case

$$
t^{|\nu|+n-p k}\left(\frac{\partial}{\partial t} x\right)^{\nu} \Phi_{k}^{ \pm}(P, t \bar{x}) \rightarrow\left(\frac{\partial}{\partial x}\right)^{\nu} \Phi_{k}^{ \pm}(P, \bar{x}) \quad \text { as } t \rightarrow 0,
$$

and hence, $(\partial / \partial x)^{\nu} \Phi_{k}^{ \pm}(P, \bar{x})=0$ when $|\nu|>p k-n$. So $\Phi_{k}^{ \pm}(P, \bar{x})$ is a homogeneous polynomial (in $\bar{x}$ ) of degree $p k-n$ in $L^{ \pm}\left(x_{0}\right)$. Then, by our previous argument, $\Phi^{ \pm}\left(\mathcal{L}_{m}, x, x_{0}\right)$ is a rational function in this domain.

Remark 3.6. The importance of the Petrovsky condition (3.33) for operators with constant coefficients is that it is both necessary and sufficient, at least when a certain stability of lacunas is required. To be precise, a (weak) lacuna $L^{ \pm}(P)$ for 
$P \in \operatorname{Hyp}(\vartheta)$ is called stable if every $\tilde{P} \in \operatorname{Hyp}(\vartheta)$ close enough to $P$ has a (weak) lacuna $L^{ \pm}(\tilde{P})$ such that $L^{ \pm}(P) \cap L^{ \pm}(\tilde{P})$ tends to $L^{ \pm}(P)$ (i.e., absorbs any compact subset of $\left.L^{ \pm}(P)\right)$ as $\tilde{P}$ tends to $P$. Petrovsky [50] proved that if $P \in \operatorname{Hyp}^{\circ}(\vartheta)$ and $\Xi^{*}$ is regular, then every stable (weak) lacuna of $\Phi^{ \pm}(P, \cdot)$ satisfies (3.33). Atiyah, Bott and Gårding [3] extended this result to the case of non-strongly hyperbolic polynomials in a somewhat weaker form: if a component $L^{ \pm}$is a regular (weak) lacuna of $\Phi_{k}^{ \pm}(P, \cdot)$ for some sufficiently large $k \in \mathbb{Z}_{+}$, then $L^{ \pm}$is stable and (3.33) holds for every point $x$ in $L^{ \pm}$. However, when $p \geq n$, there are no stable strong lacunas inside $\pm K(P, \vartheta)$. In particular, the only regular strong lacuna for all powers of $P$ is the trivial one. Applied to our case, this fact and the structure of formula (2.49) show that there are no operators $\mathcal{L}_{m}$ which have non-trivial regular strong lacunas for all values $m_{\alpha} \in \mathbb{Z}$.

Remark 3.7. Following [2], [3], we a priori restricted our attention to regular lacunas, i.e. to those lying in components of the complement of the wave front surface $\pm W$. However, the singular support of the distribution $\Phi^{ \pm}\left(\mathcal{L}, \cdot, x_{0}\right)$ may happen to fill not all of $\pm W\left(P, \vartheta, x_{0}\right)$ (i.e., the inclusion (3.1) may be proper when $\lambda=1$ ). Then some extra lacunary domains may appear in components of $V \backslash$ sing supp $\Phi^{ \pm}(\mathcal{L})$ in addition to those in $V \backslash \pm W$. Such lacunas are called irregular. For operators with constant coefficients, a few examples of this phenomenon (25], 55]) are known in higher dimensions $n>4$. However, when $n \leq 4$ or when $\Xi^{*}$ is regular enough, then we must have sing supp $\Phi^{ \pm}(P, \cdot)= \pm W(P, \vartheta)$, and all lacunas have to be regular. Imposing stability also leaves only regular lacunas, since no lacuna containing a piece of $\pm W$ can be stable. In the next section we will give an example of a hyperbolic operator with variable coefficients which admits irregular lacunas.

\section{EXAMPLES AND CONCLUding REMARKS}

The purpose of this section is to display a number of explicit examples of the theory presented so far and to illuminate some of its implications.

First, we provide an illustration for Theorem 3.2 and Corollary 3.3 It should be noted, with regard to Petrovsky's condition (3.33), that only a sparse collection of instances when (3.33) can be verified effectively is available, especially in large dimensions 8 On the other hand, there are known examples of hyperbolic polynomials (also few and far between) for which the Riesz kernel (2.19) can be evaluated explicitly in terms of classical special functions and distributions [51], 25], [55]. As shown in [30] (see also [31]), such kernels may be viewed as multidimensional analogues of Riemann-Liouville fractional integrals associated with affine homogeneous cones. Lacunary domains occurring in that case are, as a rule, irregular, and verification of Petrovsky's condition appears to be a non-trivial topological problem. The examples given below make use of both of these occurences.

Looking carefully through the literature, we could not find, after all, any examples of hyperbolic operators with variable coefficients of order $p>2$, having nontrivial (strong) lacunas. 9 The results of the present work may indicate that such operators should possess some specific properties of algebraic and algebro-geometric nature. The last example of this section is intended to make this observation more

\footnotetext{
${ }^{8}$ In fact, many of them were suggested by Petrovsky himself [50]. See also [11] and [24].

${ }^{9}$ With the possible exception of powers (or products) of second order Huygens' operators.
} 
precise. It reveals a clear link between the theory of lacunas and the problem of classification of (super-)complete commutative rings of partial differential operators, as discussed in [40], 14].

4.1. 'Trivial' examples. When $n=2$, the Petrovsky condition (3.33) is always true, while 3.32 is obviously not (with the exception of trivial cases, $m=0, p=1$ ). This amounts to the fact that $\Phi_{k}^{ \pm}(P, \cdot)$ is locally a polynomial outside $\pm W(P, \vartheta)$ for every $P \in \operatorname{Hyp}(\vartheta)$. In view of Remark 3.5 (Section 3$), \Phi^{ \pm}\left(\mathcal{L}_{m}, x, x_{0}\right)$ is then a rational function outside $\pm W\left(P, \vartheta, x_{0}\right)$ for any $\mathcal{L}_{m} \in \mathfrak{D}_{\mathrm{Hyp}}^{G, m}, m \in \mathfrak{m}_{+}$. In fact, $\Phi_{k}^{ \pm}(P, \cdot)$ is easily constructed by elementary calculation (see [2], p.174), and, hence, so is $\Phi^{ \pm}\left(\mathcal{L}_{m}, \cdot, x_{0}\right)$. Many ingenious explicit formulas for the fundamental solution of hyperbolic operators in two variables with simple dihedral symmetries can be found in the classical work of Chaundy [16].

4.2. Wave operators. The first non-trivial examples of lacunas, both mathematically interesting and physically important, occur in the case of quadratic polynomials, $P \in \operatorname{Hyp}^{\circ}(\vartheta, 2)$. Without loss of generality, we may always choose $P$ in the canonical form (2.10) with $\vartheta=( \pm 1,0, \ldots, 0)$. Clearly, $P \in \operatorname{Hyp}(\vartheta, 2)^{G}$ for any finite reflection group $G \subset O(n-1,1) \cap O(n)$. As in Example 3 (Section 2.2), we may take $G=G_{0} \times G_{1}$, where $G_{0} \subset G$ has a rank rk $G_{0} \leq n-1$ and preserves the hyperbolicity direction $\vartheta$, while $G_{1} \cong \mathbb{Z}_{2}$ is a rank one subgroup of $G$ generated by reflection in $\vartheta^{\perp}$. The corresponding wave-type operators $\mathcal{L}_{m} \in \mathfrak{D}_{\text {Hyp }}^{G, m}$ are presented in the form (2.16).

By the well-known result of M.Riesz [51] the kernel (2.19) can be evaluated explicitly. Indeed, when $\operatorname{Re} \lambda>(n-2) / 2$, the distribution $\Phi_{\lambda}^{ \pm}(P, x)$ is identified with a locally integrable function which vanishes outside the light cone $\pm K(P, \vartheta)$ and equals

$$
\Phi_{\lambda}^{ \pm}(P, x)=\frac{P(i x)^{\lambda-n / 2}}{2 \pi^{\frac{n-2}{2}} 4^{\lambda-1} \Gamma(\lambda) \Gamma(\lambda+1-n / 2)}
$$

at its interior points $x \in \pm K^{\circ}(P, \vartheta)$. For the rest values of $\lambda \in \mathbb{C}, \Phi_{\lambda}^{ \pm}(P, \cdot)$ is determined by analytical continuation.

It follows from (4.1) that $\Phi_{k}^{ \pm}(P, x)$ never vanishes inside $\pm K(P, \vartheta)$ for $k \in$ $\mathbb{Z}_{+}, k>0$, when $n$ is odd. On the other hand, for even dimensions, $\Phi_{k}^{ \pm}(P, x)$ is either a polynomial of degree $k-n / 2$ (when $k \geq n / 2$ ) or identically zero (when $k<n / 2)$ in $\pm K^{\circ}(P, \vartheta)$, i.e. there is a weak and (strong) lacuna respectively.

In the latter case, by formula (2.49), we obtain $\Phi^{ \pm}\left(\mathcal{L}_{m}\right) \equiv 0$ in $\pm K^{\circ}\left(P, \vartheta, x_{0}\right)$ for all $m \in \mathfrak{m}_{+}$such that

$$
\sum_{\alpha \in \Re_{+}} m_{\alpha}<\frac{n}{2}-1 .
$$

This result was discovered by Stellmacher [52], 53] for Coxeter groups of (splitted) $\operatorname{rank} 1, \quad G \cong \mathbb{Z}_{2} \times \cdots \times \mathbb{Z}_{2}$, and extended to the general case in [8], 9]. The expansion of the form (2.49) in this particular case was constructed in [7]. Note that verification of Petrovsky's condition (3.33) offers no difficulties for $P \in \operatorname{Hyp}(\vartheta, 2)$, though it is of course unnecessary. In fact, the local methods used in [8], [9] and based on the classical Hadamard's construction [33] are more effective in pursuing 
the problem of lacunas for second order hyperbolic operators. However, a similar explicit approach seems hardly applicable for operators of order $p \geq 3$, since fundamental solutions in that case have more complicated singularities.

4.3. Products of wave operators. The topology of Petrovsky cycles depends drastically on the parity of the space dimension. Indeed, let $\iota: \stackrel{\circ}{V^{\prime}} \rightarrow \stackrel{\circ}{V^{\prime}}, \xi \mapsto-\xi$, be the antipodal involution, and let $\sigma_{ \pm}\left(x, v ; \gamma^{\sim}\right)$ denote a relative cycle of the pair $\left(V_{\mathbb{C}}^{\prime} \backslash \Xi_{\mathbb{C}}, X_{\mathbb{C}} \backslash\left(X_{\mathbb{C}} \cap \Xi_{\mathbb{C}}\right)\right)$ associated with a given hyperbolic polynomial $P \in \operatorname{Hyp}(\vartheta)$ and some $x \in V \backslash \pm W(P, \vartheta)$ (see definitions (3.15) - (3.17)). Recall that $\sigma_{ \pm}\left(x, v ; \gamma^{\sim}\right)$ is provided by an orientation such that $(x, \xi) \omega(\xi)>0$, where $\omega(\xi)$ is a Kronecker $(n-1)$-form (3.3). In view of the absolute homogeneity of $v(\xi)$, we have $\iota\left(\sigma_{ \pm}\left(x, v ; \gamma^{\sim}\right)\right)=(-1)^{n-1} \sigma_{\mp}\left(x, v ; \gamma^{\sim}\right)$ and, hence,

$$
\iota\left(\partial \sigma_{ \pm}\left(x, v ; \gamma^{\sim}\right)\right)=(-1)^{n-1} \partial \sigma_{\mp}\left(x, v ; \gamma^{\sim}\right)
$$

for every $x$ outside $\pm W(P, \vartheta)$ and $v \in \mathfrak{V}(x, P, \vartheta)$. It follows from (4.3) that

$$
\partial\left[\sigma_{ \pm}^{*}(x)\right]=(-1)^{n-1} \partial\left[\sigma_{\mp}^{*}(x)\right],
$$

so that

$$
\partial \sigma_{ \pm}^{*}\left(x, v ; \gamma^{\sim}\right)+(-1)^{n-1} \partial \sigma_{\mp}^{*}\left(x, v ; \gamma^{\sim}\right) \in 2 \partial\left[\sigma_{ \pm}^{*}(x)\right]
$$

for all $x \in V \backslash \pm W(P, \vartheta)$. The relation (4.5) provides some interesting examples when Petrovsky's condition (3.33) holds automatically.

Suppose that a hyperplane $X \subset V^{\prime}$ dual to some point $x \in V \backslash \pm W(P, \vartheta)$ meets the real characteristic surface $\Xi(P), P \in \operatorname{Hyp}(\vartheta)$, only at the origin in $V^{\prime}$. Then, we may choose a vector field $v(\xi) \in \mathfrak{V}(x, P, \vartheta)$ in the definition of $\sigma_{ \pm}\left(x, v ; \gamma^{\sim}\right)$ in such a way that $v=0$ in some small conical neighborhood of $\stackrel{\circ}{X} \subset \stackrel{\circ}{V}^{\prime}$, and, hence, $\partial \sigma_{ \pm}\left(x, v ; \gamma^{\sim}\right)=\partial \sigma_{\mp}\left(x, v ; \gamma^{\sim}\right)$ in that case. When $n$ is even, this implies the vanishing of $\partial\left[\sigma_{ \pm}^{*}(x)\right]$ in view of (4.5) :

$$
X \cap \Xi=\{0\} \quad \Rightarrow \quad \partial\left[\sigma_{ \pm}^{*}(x)\right]=0 \quad \text { in } \quad H_{n-2}\left(X_{\mathbb{C}}^{*} \backslash\left(X_{\mathbb{C}}^{*} \cap \Xi_{\mathbb{C}}^{*}\right) ; \mathbb{C}\right) .
$$

The condition (4.6) applies to the product of wave polynomials with different light velocities. Indeed, put

$$
P(\zeta):=\prod_{k=1}^{l}\left(-\left(\zeta^{1} / c_{k}\right)^{2}+\left(\zeta^{2}\right)^{2}+\cdots+\left(\zeta^{n}\right)^{2}\right),
$$

where $0<c_{1} \leq c_{2} \leq \cdots \leq c_{l-1} \leq c_{l}$ are arbitrary real constants. Then, $P(\zeta)$ is both hyperbolic in direction $\vartheta=( \pm 1,0, \ldots, 0)$, and invariant with respect to any group $G$ generated by reflections preserving this direction. The corresponding operators $\mathcal{L}_{m} \in \mathfrak{D}_{\text {Hyp }}^{G, m}$ are written in the form

$$
\mathcal{L}_{m}=\prod_{k=1}^{l}\left(\frac{1}{c_{k}^{2}} \frac{\partial^{2}}{\partial x_{1}^{2}}-\frac{\partial^{2}}{\partial x_{2}^{2}}-\cdots-\frac{\partial^{2}}{\partial x_{n}^{2}}+\sum_{\alpha \in \Re_{+}} \frac{m_{\alpha}\left(m_{\alpha}+1\right)(\alpha, \alpha)}{(\alpha, x)^{2}}\right) .
$$

The wave front surface $\pm W(P, \vartheta)$ consists of $l$ telescoping conics and splits the space $V$ into $2 l+1$ connected components, one of them being the outer component $V \backslash \pm K(P, \vartheta)$ and one being the 'most inner' convex cone $\pm K_{\text {in }}(P, \vartheta)$ inside $\pm K(P, \vartheta)$. It is easy to see that $\stackrel{\circ}{X} \cap \Xi$ is empty for every point $x \in \pm K_{\text {in }}(P, \vartheta)$. Hence, when $n$ is even, the condition (4.6) holds for these points. By Corollary [3.3, we conclude that any open subset $L^{ \pm}\left(x_{0}\right)$ in $\pm K_{\text {in }}\left(P, \vartheta, x_{0}\right)$ lying outside the fixed singularity 


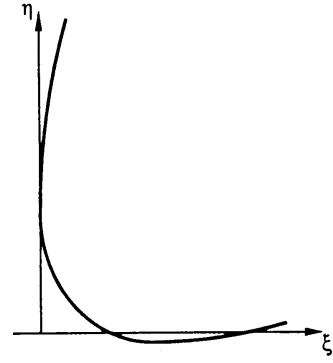

(a)

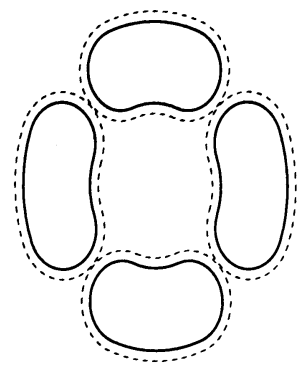

(b)

Figure 2. Petrovsky's example I

locus $\bigcup \alpha^{\perp}, \alpha \in \Re$, is a regular strong lacuna of the operator [4.8), provided $n$ is even, and

$$
\sum_{\alpha \in \Re_{+}} m_{\alpha}<\frac{n}{2 l}-1 .
$$

Note that, when the $c_{k}$ are all distinct, the polynomial (4.7) is strongly hyperbolic. In that case, we may generalize example (4.7). Indeed, let $n, p$ both be even, and assume that $P \in \operatorname{Hyp}^{\circ}(\vartheta)$ is strongly hyperbolic. If every part of $\Xi(P)$ bounds a convex cone in $V^{\prime}$, then (4.6) still applies and Petrovsky's condition (3.33) holds for any $x$ inside the 'most inner' component $\pm K_{\text {in }}(P, \vartheta)$ of $\pm K(P, \vartheta)$. This result was already known to Petrovsky ([50], p. 345). A simple proof based on Gel'fand's version [29] of the Herglotz-Petrovsky formulas can be found in 11].

The existence of lacuna in the 'most inner' component of the propagation cone implies the absence of wave diffusion, a phenomenon which, according to [50], may be viewed as an analog of Huygens' principle for higher order hyperbolic operators. A detailed study of the Cauchy problem for the operators (4.7) has been carried out in [24. Simple geometric arguments used here to show (4.6) are essentially due to $[2$.

4.4. Petrovsky's examples. As shown in [3], the validity of Petrovsky's condition (3.33) in even dimensions $n>2$ amounts to the fact that the real intersection $\Xi^{*} \cap X^{*} \subset \mathbb{R P}^{n-1}$ of the projective algebraic surface $\Xi^{*}$ with a hyperplane $X^{*}$ is homologous to zero in their complex intersection $\Xi_{\mathbb{C}}^{*} \cap X_{\mathbb{C}}^{*} \subset \mathbb{C P}^{n-1}$, at least when the latter has no singular points. Condition (4.6) is a trivial manifestation of this situation. Petrovsky ([50], pp. 349-350) constructed the following non-trivial example when $p=4$.

Consider a real hyperbola $H(\xi, \eta)=0$ located in the coordinate plane $(\xi, \eta)$ as shown in Fig. 2(a). It meets a positive horizontal semi-axis at two distinct points while touching the vertical one at some point $\left(0, \eta_{0}\right), \eta_{0}>0$. We assume $H(0,0)<0$.

The homogeneous polynomial defined by

$$
\begin{aligned}
P(\zeta) & :=\zeta_{n}^{4}\left[H\left(\left(\zeta_{1} / \zeta_{n}\right)^{2}+\cdots+\left(\zeta_{n-2} / \zeta_{n}\right)^{2},\left(\zeta_{n-1} / \zeta_{n}\right)^{2}\right)-b \varepsilon^{3}\right] \\
& -\varepsilon \zeta_{n-1} \zeta_{n}\left(\zeta_{1}^{2}+\cdots+\zeta_{n-3}^{2}-\zeta_{n-2}^{2}\right)+a \varepsilon^{2}\left(\zeta_{1}^{2}+\cdots+\zeta_{n-3}^{2}-\zeta_{n-2}^{2}\right)^{2}
\end{aligned}
$$




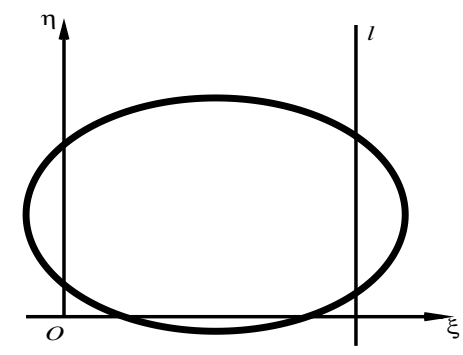

Figure 3. Petrovsky's example II

is strongly hyperbolic, when $a, b$ and $\varepsilon$ are sufficiently small (positive or negative) real constants. Then, the projective surface $\Xi^{*}$ characteristic to (4.10) consists of two non-intersecting ovals embedded one into another with no singular points. Petrovsky showed that a real intersection of $\Xi^{*}$ with a hyperplane $X^{*}: \zeta_{n-1}=0$ is homologous to zero in $\Xi_{\mathbb{C}}^{*} \cap X_{\mathbb{C}}^{*}$. (When $n=4$, the surface $\Xi^{*} \cap X^{*}$ consists of four properly oriented ovals in $\mathbb{R}^{2}$ shown schematically in Fig. 2(b).) In even dimensions $n \geq 4$, this implies the validity of (3.33) for the component of $\pm K(P, \vartheta) \backslash$ $\pm W(P, \vartheta)$ containing the point $x \in V$ dual to $X^{*}$.

The polynomial (4.10) is invariant under any reflection group acting in the space of variables $\left(\zeta_{1}, \ldots, \zeta_{n-3}\right)$. For every such group $G$ the hyperbolic operators $\mathcal{L}_{m} \in$ $\mathfrak{D}_{\text {Hyp }}^{G, m}$ with (4.10) as a principal symbol may have regular lacunas $L^{ \pm}\left(x_{0}\right)$ in the component of $\pm K\left(P, \vartheta, x_{0}\right) \backslash \pm W\left(P, \vartheta, x_{0}\right)$ which contains the point $x$ dual to $X$. By Corollary 3.3 , this happens when $m \in \mathfrak{m}_{+}, n \geq 4$ is even, and

$$
\sum_{\alpha \in \Re_{+}} m_{\alpha}<\frac{n}{4}-1
$$

The relation (3.33) may be valid not only in even but in odd dimensions as well. The following sufficient condition is due to Atiyah, Bott and Gårding (see [2, Theorem 6.27).

Let $n \geq 3$ be odd and let $P_{x}$ be the restriction of $P \in \operatorname{Hyp}(\vartheta)$ to a hyperplane $X_{\mathbb{C}} \subset V_{\mathbb{C}}^{\prime}$. If $x \notin \pm W(P, \vartheta)$ and there is a $\vartheta_{x} \in X$ such that $P_{x} \in \operatorname{Hyp}\left(\vartheta_{x}\right)$ and at least one of the sets $\pm \Gamma_{\xi}\left(P_{x}, \vartheta_{x}\right) \cap \Gamma_{\xi}(P, \vartheta)$ is never empty for $\xi \in \stackrel{\circ}{X}$, then the Petrovsky condition holds. The proof of this result is based on another example suggested by Petrovsky ([50], p. 348). Consider an ellipse $H(\xi, \eta)=0$ in the coordinate plane $(\xi, \eta)$, crossing both (positive) semi-axes at two distinct points (see Fig. 3), and define the following polynomial:

$$
P(\zeta):=\zeta_{n}^{4} H\left(\left(\zeta_{1} / \zeta_{n}\right)^{2}+\cdots+\left(\zeta_{n-2} / \zeta_{n}\right)^{2},\left(\zeta_{n-1} / \zeta_{n}\right)^{2}\right)
$$

The characteristic surface $\Xi^{*}$ of $P$ consists of two real ovals contained in one another, and $P(\zeta)$ is hyperbolic with regard to any $\vartheta^{*}$ in $\Gamma^{*}$, the domain bounded by the inner oval. Let $X^{*}$ be a hyperplane produced by revolving a line $l$ around the $\zeta_{n-1}$-axis. The intersection $\Xi^{*} \cap X^{*}$ consists of two ovals in one another, the restriction $P_{x}$ being hyperbolic with respect to $\vartheta_{x}^{*}$, a point inside the smaller of them. It is not difficult to see that $\pm \Gamma_{\xi}^{*}(P, \vartheta) \cap X^{*}$ are all at least half-spaces for $\xi \in X$, and then, by the convexity of $\Gamma_{\xi}^{*}\left(P_{x}, \vartheta_{x}\right)$ in $X^{*}$, at least one of the sets $\pm \Gamma_{\xi}^{*}\left(P_{x}, \vartheta_{x}\right) \cap \Gamma_{\xi}^{*}(P, \vartheta)$ is never empty. When $n \geq 3$ is odd, this implies the 
validity of (3.33) inside the components $L^{ \pm}$containing the point $x \in \pm W(P, \vartheta)$ dual to $X^{*}$. The Petrovsky polynomial $P(\zeta)$ is invariant under any reflection group $G$ acting in the space of variables $\left(\zeta_{1}, \ldots, \zeta_{n-2}\right)$, and hence the related operators $\mathcal{L}_{m} \in \mathfrak{D}_{\text {Hyp }}^{G, m}$ possess non-trivial lacunas $L^{ \pm}\left(x_{0}\right)$ when $n$ is odd and (4.11) holds.

4.5. Irregular lacunas and the strong Huygens' principle. The absence of diffusion of waves is one possible generalization of Huygens' principle to higher order hyperbolic operators, $p>2$. In the case of constant coefficients this phenomenon has a purely topological nature.

Another analog of this notion (which is apparently related to differential operators with specific algebraic properties, e. g., with a large symmetry group) consists in the fact that the support of $\Phi(\mathcal{L}, \cdot)$ may fill a part of some (real) surface $S$ of positive codimension $\operatorname{codim} S \geq 1$ in $V$. For $\mathcal{L}=P(D), P \in \operatorname{Hyp}^{\circ}(\vartheta)$, such a situation never happens, with the sole exception of wave operators (0.1) in even dimensions, $n \geq 4$ (see [11]). However, there are examples (25], [55]) with multiple characteristics $(p>2)$ when Huygens' principle may hold even in the stronger form: codim $\operatorname{supp} \Phi(\mathcal{L}, \cdot)>1$. This corresponds to occurence of irregular lacunas in the propagation cone of $\mathcal{L}$ (see Remark 3.7 Section 3).

Here, we give an example of a hyperbolic operator with variable coefficients which satisfies the strong Huygens' principle.

Let $V=\bigoplus V_{j}, j=0,1,2$, with $\operatorname{dim} V_{0}=3, \operatorname{dim} V_{1}=n_{1}, \operatorname{dim} V_{2}=n_{2}, n_{1,2}>$ 0 , and consider the following polynomial $P(\zeta) \in \mathbb{C}\left[V^{\prime}\right]$

$$
\begin{aligned}
P(\zeta):= & \zeta_{01} \zeta_{02} \zeta_{03}-\frac{1}{4} \zeta_{02}\left(\zeta_{11}^{2}+\zeta_{12}^{2}+\cdots+\zeta_{1 n_{1}}^{2}\right) \\
& -\frac{1}{4} \zeta_{01}\left(\zeta_{21}^{2}+\zeta_{22}^{2}+\cdots+\zeta_{2 n_{2}}^{2}\right)
\end{aligned}
$$

written in some fixed coordinate bases $\left\{\zeta_{j k}\right\}_{k=1}^{n_{j}}$ in $V_{j}^{\prime} \cong V_{j}, j=0,1,2$. It is easy to see that (4.13) is hyperbolic with hyperbolicity cone

$$
\Gamma(P, \cdot):=\left\{\zeta \in V^{\prime} \mid P(\zeta)>0, \zeta_{01}>0, \zeta_{02}>0\right\}
$$

and the propagation cone

$$
K(P, \cdot):=\left\{x \in V \mid Q_{1}(x) \geq 0, Q_{2}(x) \geq 0, x_{03} \geq 0\right\},
$$

where $Q_{1}(x):=x_{01} x_{03}-\sum_{k=1}^{n_{1}} x_{1 k}^{2}, Q_{2}(x):=x_{02} x_{03}-\sum_{k=1}^{n_{2}} x_{2 k}^{2}$.

The study of the support structure of the kernel $\Phi_{\lambda}(\mathcal{L}, \cdot)$ can be carried out along the same lines as in [55]. It turns out that when $n_{1}$ and $n_{2}$ are both even and $k \leq \frac{1}{2} \min \left(n_{1}, n_{2}\right)$ is a positive integer, $k \in \mathbb{Z}_{+}, \operatorname{supp} \Phi_{k}(P, \cdot)$ constitutes a (semi-)algebraic surface in $V$ defined by

$$
S_{0}:=\left\{x \in V \mid Q_{1}(x)=0, Q_{2}(x)=0, x_{03} \geq 0\right\} .
$$

Take $G=G_{1} \times G_{2} \subset O(V)$, a direct product of finite reflection groups $G_{1} \subset$ $O\left(V_{1}\right)$ and $G_{2} \subset O\left(V_{2}\right)$ respectively, $\operatorname{rk} G \leq n_{1}+n_{2}$, and define

$$
\begin{aligned}
\mathcal{L}_{m}:= & P(D)+\frac{i}{4}\left(\sum_{\alpha \in \Re_{1_{+}}} \frac{m_{\alpha}\left(m_{\alpha}+1\right)(\alpha, \alpha)}{(\alpha, x)^{2}}\right) \frac{\partial}{\partial x_{02}} \\
& +\frac{i}{4}\left(\sum_{\beta \in \Re_{2_{+}}} \frac{m_{\beta}\left(m_{\beta}+1\right)(\beta, \beta)}{(\beta, x)^{2}}\right) \frac{\partial}{\partial x_{01}}
\end{aligned}
$$

with $\Re_{1}:=\Re\left(G_{1}\right), \Re_{2}:=\Re\left(G_{2}\right)$ and $m \in M(G)$. 
It follows from Theorem 2.17 that

$$
\begin{aligned}
\operatorname{supp} \Phi\left(\mathcal{L}_{m}\right) \subseteq S_{x_{0}}:=\left\{x \in V \mid Q_{1}\left(x-x_{0}\right)=0, Q_{2}\left(x-x_{0}\right)=0,\right. & \\
& \left.x_{03}-x_{03}^{0} \geq 0\right\},
\end{aligned}
$$

when $m \in \mathfrak{m}_{+}, n_{1}, n_{2}$ are even and

$$
\sum_{\alpha \in \Re_{1+}} m_{\alpha}+\sum_{\beta \in \Re_{2+}} m_{\beta}<\frac{1}{2} \min \left(n_{1}, n_{2}\right)-1 .
$$

Thus, for (4.16) Huygens' principle holds in the strong form.

4.6. Closing remarks. In the present paper we have studied a class $\mathfrak{D}_{\mathrm{Hyp}}^{G, m}$ of hyperbolic differential operators with singular coefficients for which fundamental solutions can be constructed explicitly in terms of Herglotz-Petrovsky-Leray type Abelian integrals (Theorem 3.2). The structure of these integral formulas allows the existence of non-trivial (strong) lacunas, provided the Petrovsky topological condition holds for the symbol. For any (fixed) $m \in M(G), \mathfrak{D}_{\text {Hyp }}^{G, m}$ is embedded in a commutative ring of partial differential operators $\mathfrak{D}^{G, m}$ isomorphic to the free $\mathbb{C}$-algebra of $G$-invariant polynomials $\mathbb{C}\left[V^{\prime}\right]^{G}$ (cf. Theorem 1.7). It turns out that in case of integer multiplicities, $m \in \mathfrak{m}_{+}, \mathfrak{D}^{G, m}$ admits a non-trivial commutative extension $\mathfrak{D}^{G, m} \rightarrow \mathfrak{D}_{\sim}^{G, m}$ isomorphic to the ring $\mathbb{C}\left[V^{\prime}\right]_{\sim}^{G}$ of quasi-invariants of the Coxeter group $G$, the latter being defined by (see [14])

$\mathbb{C}\left[V^{\prime}\right]_{\sim}^{G}:=\left\{P \in \mathbb{C}\left[V^{\prime}\right] \mid \partial_{\alpha} P=\partial_{\alpha}^{3} P=\cdots=\partial_{\alpha}^{2 m_{\alpha}-1} P=0\right.$ on $\left.(\alpha, \zeta)=0, \alpha \in \Re\right\}$.

Note that $\mathbb{C}\left[V^{\prime}\right]_{\sim}^{G}$ is a supercomplete ring in the sense that the (minimal) number of generators of $\mathbb{C}\left[V^{\prime}\right]_{\sim}^{G}$ (as a $\mathbb{C}$-algebra) exceeds its (Krull) dimension $n=\operatorname{dim} V$.

Algebraically, the existence of extra non-invariant differential operators in the centralizer $\mathcal{Z}\left(\mathfrak{D}^{G, m}\right)$ of $\mathfrak{D}^{G, m}$ in $\mathfrak{D}$ is closely related to the existence of lacunary hyperbolic operators in $\mathfrak{D}_{\text {Hyp }}^{G, m}$.

To make this observation precise, we will construct one such operator explicitly by evaluating the Riesz kernel of a specific $G$-invariant hyperbolic operator in $\mathfrak{D}^{G, m}$ at a non-trivial singular point $\lambda \in \mathbb{C}$ (for more details see [4]).

Let $P(\zeta)=\prod_{\alpha \in \Re}(\alpha, \zeta)$ be a discriminant of a Coxeter group $G$. As discussed in Example 2.9 (Section 2.2), $P(\zeta) \in \operatorname{Hyp}(\vartheta)^{G}$ with $\vartheta \in \Gamma_{+}(G)$. Define $\mathcal{L}_{m}:=$ $\operatorname{Res} \nabla_{m} P(\zeta) \in \mathfrak{D}_{\text {Hyp }}^{G, m}$ for $m \in \mathfrak{m}_{+}$. According to Theorem 2.17 $\mathcal{L}_{m}$ is properly hyperbolic and its Riesz kernel is given by formula (2.48).

When $\lambda \in-M-\frac{1}{2} \mathbb{Z}_{+}$, the distribution $\Phi_{\lambda}\left(\mathcal{L}_{m}, x, x_{0}\right)$ has a point support $\operatorname{supp} \Phi_{\lambda}\left(\mathcal{L}_{m}, \cdot, x_{0}\right)=\left\{x_{0}\right\}$, and hence there is a (degenerate) regular lacuna inside $K\left(P, x, x_{0}\right)$. It follows from (2.48) that $\Phi_{\lambda}\left(\mathcal{L}_{m}, x, x_{0}\right)=\mathcal{L}_{m}^{\lambda}\left[\delta\left(x-x_{0}\right)\right]$ for $\lambda \in$ $-M-\mathbb{Z}_{+}$, and

$$
\Phi_{\lambda}\left(\mathcal{L}_{m}\right)=\sum_{k=0}^{M}(-1)^{k} \frac{(\lambda)_{k}}{k !} \operatorname{ad}^{k}\left(\mathcal{L}_{m}, \mathcal{L}_{0}\right)\left[\Theta_{m}(x)\right] \pi(D)^{-2 k-2 \lambda}\left[\delta\left(x-x_{0}\right)\right]
$$

for $\lambda \in-M-\frac{1}{2} \mathbb{Z}_{+}^{\text {odd }}$, where $\pi(D):=\prod_{\alpha \in \Re_{+}}(\alpha, D)$.

The differential operator

$$
\tilde{\mathcal{L}}_{m}:=\sum_{k=0}^{M}(-1)^{k} \frac{(-M-1 / 2)_{k}}{k !} \operatorname{ad}^{k}\left(\mathcal{L}_{m}, \mathcal{L}_{0}\right)\left[\theta_{m}(x)\right] \pi(D)^{2(M-k)+1} \theta_{m}(x)^{-1}
$$


associated with the integral kernel (4.18) has a skew-invariant principal symbol $\pi(\zeta)^{2 M+1}$, while commuting with any element in $\mathfrak{D}^{G, m}$. Indeed, by the uniqueness of the Riesz kernel $\Phi_{\lambda}\left(\mathcal{L}_{m}, x, x_{0}\right)$, we have the duality property

$$
Q\left[\Phi_{\lambda}\left(\mathcal{L}_{m}, \cdot, x_{0}\right)\right](x)=Q^{*}\left[\Phi_{\lambda}\left(\mathcal{L}_{m}, x, \cdot\right)\right]\left(x_{0}\right)
$$

for any $Q \in \mathfrak{D}$ commuting with $\mathcal{L}_{m}$. For singular values $\lambda \in-M-\frac{1}{2} \mathbb{Z}_{+}$, equation (4.20) boils down to the necessary commutativity relation $\left[\tilde{\mathcal{L}}_{m}, Q\right]=0$. Thus, $\tilde{\mathcal{L}}_{m} \in \mathcal{Z}\left(\mathfrak{D}^{G, m}\right) \backslash \mathfrak{D}^{G, m}$.

With regard to the above example, it seems tempting to adopt some (possibly, very tentative) hypothesis on algebro-geometric characterization of lacunary hyperbolic operators with variable coefficients.

Conjecture 4.1. Let $\mathcal{L}:=P(D)+\sum a_{\nu}(x) D^{\nu}$ be a hyperbolic differential operator defined in some open part $\Omega \subset V$ with a constant principal symbol $P \in \operatorname{Hyp}(\vartheta)$ and smooth lower order coefficients $a_{\nu}(x) \in \mathcal{C}^{\infty}(\Omega)$. Suppose that $\Phi\left(\mathcal{L}, \cdot, x_{0}\right)$ has a non-trivial (strong) lacuna inside $K\left(P, \vartheta, x_{0}\right)$ at every $x_{0} \in \Omega$. Then, there exists a commutative ring $\mathfrak{D}_{\mathcal{L}}$ of partial differential operators such that $\mathcal{L} \in \mathfrak{D}_{\mathcal{L}}$, and $\mathfrak{D}_{\mathcal{L}}$ is isomorphic to some supercomplete subalgebra in $\mathbb{C}\left[V^{\prime}\right]$.

In the case of second order wave-type operators this conjecture has been verified for all known classes of lacunary (i.e., Huygens) operators (see [10, [9], [5], [59]).

\section{REFERENCES}

1. L. Asgeirsson, Some hints on Huygens' principle and Hadamard's conjecture, Comm. Pure Appl. Math., 9 (1956), 307-326. MR 18:487d

2. M.F. Atiyah, R. Bott, and L. Gårding, Lacunas for hyperbolic differential operators with constant coefficients I, Acta Math., 124 (1970), 109-189. MR 57:10252a

3. M.F. Atiyah, R. Bott, and L. Gårding, Lacunas for hyperbolic differential operators with constant coefficients II, Acta Math., 131 (1973), 145-206. MR 57:10252b

4. Yu.Yu. Berest, Lacunae of hyperbolic Riesz kernels and commutative rings of partial differential operators, Lett. Math. Phys. 41 (1997), 227-235. MR 98k:58103

5. Yu.Yu. Berest, Solution of a restricted Hadamard problem on Minkowski spaces, Comm. Pure Appl. Math., 50 (1997), 1019-1052. MR 99c:35135]

6. Yu.Yu. Berest, The theory of lacunas and quantum integrable systems, in Proceedings of the Workshop The Calogero-Moser-Sutherland Model (J.-F. van Diejen and L. Vinet, Eds.), CRM Series in Mathematical Physics, Springer-Verlag (1998), to appear.

7. Yu.Yu. Berest, and Yu.A. Molchanov, Fundamental solutions for partial differential operators with reflection group invariance, J. Math. Phys. 36(8) (1995), 4324-4339. MR 96c:35005

8. Yu.Yu. Berest, and A.P. Veselov, Huygens' principle and Coxeter groups, Russian Math. Surveys, 48(2) (1993), 183-184. MR 94i:35110

9. Yu.Yu. Berest, and A.P. Veselov, Hadamard's problem and Coxeter groups: new examples of Huygens' equations, Funct. Anal. Appl., 28 (1994), 3-12. MR 95h:58131

10. Yu.Yu. Berest, and A.P. Veselov, Huygens' principle and integrability, Russian Math. Surveys, 49(6) (1994), 5-77. MR 96a:35003

11. V.A. Borovikov, Some sufficient conditions for the absence of lacunas, Mat. Sbornik, 55 (3) (1961), 237-254. (Russian) MR 25:5284

12. L. Boutet de Monvel, Lacunas and transmissions in Seminar on Singularities of Solutions of Linear Partial Differential Equations, Ann. of Math. Stud. 91, Princeton Univ. Press, Princeton, 1978, pp. 209-218. MR 80k:58092

13. J. Chaillou, Les Polynômes Différentiels Hyperboliques et Leurs Perturbations Singulières, Gauthiers-Villars, Paris, 1973. MR 57:3651

14. O.A. Chalykh, and A.P. Veselov, Commutative rings of partial differential operators and Lie algebras, Comm. Math. Phys., 126 (1990), 597-611. MR 91g:58106

15. O.A. Chalykh, and A.P. Veselov, Integrability in the theory of the Schrödinger operator and harmonic analysis, Comm. Math. Phys., 152 (1993), 29-40. MR 94a:58160 
16. T.W. Chaundy, Hypergeometric partial differential equations, Quart. J. Math., Oxford Ser. 6 (1935), 288-303; 7 (1936), 305-315; 8 (1937), 280-302; 9 (1938), 234-240; 10 (1939), 219-240; 11 (1940), 101-110. MR 1:119g

17. R. Courant, and D. Hilbert, Methods of Mathematical Physics II, Interscience Publ., N. Y., 1962. MR 25:4216

18. G.F.D. Duff, Singularities, supports and lacunas, in Advances in Microlocal Analysis, NATO Adv. Sci. Inst. Ser. C: Math. Phys. Sci., 168, Reidel, Dordrecht-Boston, 1986, pp. 73-133. MR 87j:35003

19. J.J. Duistermaat, and L. Hörmander, Fourier integral operators II, Acta Math., 128 (1972), 183-269. MR 52:9300

20. C.F. Dunkl, Differential-difference operators associated to reflection groups, Trans. AMS, 311 (1989), 167-183. MR 90k:33027

21. C.F. Dunkl, Hankel transforms associated to finite reflection groups, Contemporary Math., 138 (1992), 123-138. MR 94g:33011

22. F.G. Friedlander, The Wave Equation on a Curved Space-Time, Cambridge Univ. Press, Cambridge, 1975. MR 57:889

23. A.M. Gabrielov, and A.P. Palamodov, Huygens' principle and its generalizations in I.G.Petrovsky "Selected Works", Nauka, Moscow, 1986, pp. 449-456. MR 88f:01059 English transl. in: I. G. Petrovsky, "Selected Works", Part I, Classics of Soviet Mathematics 5, Gordon and Breach Publ., Amsterdam, 1996, pp. 485-495.

24. S.A. Gal'pern, and V.E. Kondrashov, The Cauchy problem for differential operators decomposing into wave factors, Trans. Moscow Math. Soc., 16 (1967), 117-145. MR 37:585

25. L. Gårding, The solution of Cauchy's problem for two totally hyperbolic linear differential equations by means of Riesz integrals, Ann. Math., 48 (1947), 785-826. MR 9:240a

26. L. Gårding, Linear hyperbolic partial differential equations with constant coefficients, Acta Math., 85 (1950), 1-62. MR 12:831g

27. L. Gårding, Sharp fronts of paired oscillatory integrals, Publ. RIMS, Kyoto Univer., 12 (1977), 53-68 (Corrections: ibid., 13 (1977), 821). MR 57:10253a, MR 57:10253b

28. L. Gårding, Singularities in Linear Wave Propagation, Lecture Notes in Math, 1241, Springer-Verlag, Berlin, 1987. MR 88k:35002

29. I.M. Gel'fand, and G.E. Shilov, Generalized Functions I, Academic Press, New York, 1964. MR 29:3869

30. S.G. Gindikin, Analysis in homogeneous domains, Russ. Math. Surveys, 19(4) (1964), 1-89. MR 30:2167

31. S.G. Gindikin, Tube Domains and the Cauchy Problem, Transl. Math. Monographs, 111, Amer. Math. Soc., Providence, RI, 1992. MR 94d:35028

32. P. Günther, Huygens' Principle and Hyperbolic Equations, Academic Press, Boston, 1988. MR 89j:35077

33. J. Hadamard, Lectures on Cauchy's Problem in Linear Partial Differential Equations, Yale Univ. Press, New Haven, 1923 (reprinted by Dover, N. Y., 1956). MR 14:474f

34. J. Hadamard, The problem of diffusion of waves, Annals of Math., 43(3) (1942), 510-522. MR 4:45a

35. G.J. Heckman, A remark on the Dunkl differential-difference operators, Progr. in Math., 101 Birkhäuser, Boston, 1991, pp. 181-191. MR 94c:20075

36. G.J. Heckman, and H.Schlichtkrull, Harmonic Analysis and Special Functions on Symmetric Spaces, Academic Press, San Diego, 1994. MR 96j:22019

37. S. Helgason, Wave equations on homogeneous spaces, Lecture Notes in Math., 1077 (1984), 252-287. MR 86c:58141]

38. L. Hörmander, Fourier integral operators I, Acta Math., 127 (1971), 79-183. MR 52:9299

39. L. Hörmander, The Analysis of Linear Partial Differential Operators, I-IV, Springer-Verlag, Berlin, 1983-85. MR 85g:35002a MR 85g:35002b MR 87d:35002a |MR 87d:35002b

40. I.M. Krichever, Methods of algebraic geometry in the theory of nonlinear equations, Russian Math. Surveys, 32(6) (1977), 198-220. MR 58:24353

41. J.E. Lagnese, A solution of Hadamard's problem for a rectricted class of operators, Proc. Amer. Math. Soc., 19 (1968), 981-988. MR 37:6581

42. A. Lax, On Cauchy's problem for partial differential equations with multiple characteristics, Comm. Pure Appl. Math., 9 (1956), 135-169. MR 18:397b 
43. H. Lewy, The wave equation as limit of hyperbolic equations of higher order, Comm. Pure Appl. Math., 17 (1965), 5-16. MR 30:5059

44. M. Mathisson, Le probléme de M. Hadamard relatif a la diffusion des ondes, Acta Math., 71 (1939), 249-282. [MR 1:120e|

45. R.G. McLenaghan, Huygens' principle, Ann. Inst. Henri Poincaré Sect. A 37(3) (1982), 211236. MR 84e:35091

46. W. Nuij, A note on hyperbolic polynomials, Math. Scand., 23 (1968), 69-72. MR 40:3368

47. M.A. Olshanetsky, and A.M. Perelomov, Quantum integrable systems related to Lie algebras, Phys. Reps., 94 (1983), 313-404. MR 84k:81007

48. E. Opdam Root systems and hypergeometric functions III, IV, Compositio Math., 67 (1988), 21-49, 191-209. MR 90k:17012; MR 90c:58079

49. E. Opdam, Dunkl operators, Bessel functions, and the discriminant of a finite Coxeter group, Compositio Math., 85 (1993), 333-373. MR 95j:33044

50. I.G. Petrowsky, On the diffusion of waves and the lacunas for hyperbolic equations, Mat. Sbornik, 17(59) (1945), 289-370. (English; Russian summary) MR 8:79a

51. M. Riesz, L'intégrale de Riemann-Liouville et le problème de Cauchy, Acta Math., 81 (1949), 1-223. MR 10:713c

52. K.L. Stellmacher, Ein Beispiel einer Huyghensschen Differentialgleichung, Nachr. Akad. Wiss. Göttingen, Math.-Phys. K1, IIa, 10 (1953), 133-138. MR 15:710e

53. K.L. Stellmacher, Ein Klasse Huyghenscher Differentialgleichung und ihre Integration, Math. Ann., 130(3) (1955), 219-233. MR 17:494e

54. S.L. Svensson, Necessary and sufficient conditions for the hyperbolicity of polynomials with hyperbolic principal part, Arkiv Math., 8 (1969), 145-162 MR 42:6421

55. B.R. Vainberg, S.G. Gindikin, A strengthened Huygens' principle for a class of differential operators with constant coefficients, Trans. Moscow Math. Soc., 16 (1967), 163-196. MR 37:3176

56. V.A. Vassiliev, Sharpness and local Petrovsky condition for strictly hyperbolic operators with constant coefficients, Izv. Akad. Nauk SSSR Ser. Mat. 50(2) (1986), 243-284; English transl., Math. USSR Izv. 28 (1987), 233-273. MR 88a:58193

57. V.A. Vassiliev, Ramified Integrals, Singularities and Lacunas, Mathematics and its Appl., 315, Kluwer Acad. Publ., 1995. MR 96h:32052

58. A.P. Veselov, K.L. Styrkas, and O.A. Chalykh, Algebraic integrability for the Schrödinger operator and reflection groups, Theor. Math. Phys. 94 (1993), 182-197. MR 94j:35151

59. A.P. Veselov, M.V. Feigin, and O.A. Chalykh, New integrable generalizations of quantum Calogero-Moser problem, Usp. Mat. Nauk, 51(3) (1996), 185-186; English transl. in Russian Math. Surveys 57 (1996), no. 3. MR 97e:58122

Department of Mathematics, University of California, Berkeley, California 94720 Current address: Department of Mathematics, Cornell University, Ithaca, New York 148534201

E-mail address: berest@math.cornell.edu 